International Journal of Modern Physics B Vol. 28, No. 14 (2014) 1430006 (34 pages)

(C) The Authors

DOI: $10.1142 / \mathrm{S} 0217979214300060$

\title{
Complex and unexpected dynamics in simple genetic regulatory networks*
}

\author{
Yanika Borg \\ Department of Mathematics and Department of Biochemical Engineering, \\ University College London, Gower Street, \\ London, WC1E 6BT, United Kingdom \\ yanika.borg.11@ucl.ac.uk \\ Ekkehard Ullner \\ Department of Physics (SUPA), \\ Institute for Complex Systems and Mathematical Biology, \\ (ICSMB) and Institute of Medical Sciences, \\ University of Aberdeen, Aberdeen, AB24 3UE, United Kingdom \\ e.ullner@abdn.ac.uk \\ Afnan Alagha ${ }^{\dagger}$ and Ahmed Alsaedi ${ }^{\ddagger}$ \\ Nonlinear Analysis and Applied Mathematics Research Group (NAAM), \\ Department of Mathematics, King Abdulaziz University, Jeddah, Saudi Arabia \\ †fofoalagha_333@hotmail.com \\ $\ddagger$ aalsaedi@hotmail.com \\ Darren Nesbeth \\ Department of Biochemical Engineering, University College London, \\ Gower Street London, WC1E 6BT, United Kingdom \\ d.nesbeth@ucl.ac.uk \\ Alexey Zaikin \\ Institute of Women's Health and Department of Mathematics, \\ University College London, Gower Street, London, \\ WC1E 6BT, United Kingdom \\ Lobachevsky State University of Nizhniy Novgorod, Nizhniy Novgorod, Russia \\ alexey.zaikin@ucl.ac.uk
}

Received 8 February 2014

Accepted 16 February 2014

Published 25 March 2014

\begin{abstract}
*This is an Open Access article published by World Scientific Publishing Company. It is distributed under the terms of the Creative Commons Attribution 3.0 (CC-BY) License. Further distribution of this work is permitted, provided the original work is properly cited.
\end{abstract}


One aim of synthetic biology is to construct increasingly complex genetic networks from interconnected simpler ones to address challenges in medicine and biotechnology. However, as systems increase in size and complexity, emergent properties lead to unexpected and complex dynamics due to nonlinear and nonequilibrium properties from component interactions. We focus on four different studies of biological systems which exhibit complex and unexpected dynamics. Using simple synthetic genetic networks, small and large populations of phase-coupled quorum sensing repressilators, Goodwin oscillators, and bistable switches, we review how coupled and stochastic components can result in clustering, chaos, noise-induced coherence and speed-dependent decision making. A system of repressilators exhibits oscillations, limit cycles, steady states or chaos depending on the nature and strength of the coupling mechanism. In large repressilator networks, rich dynamics can also be exhibited, such as clustering and chaos. In populations of Goodwin oscillators, noise can induce coherent oscillations. In bistable systems, the speed with which incoming external signals reach steady state can bias the network towards particular attractors. These studies showcase the range of dynamical behavior that simple synthetic genetic networks can exhibit. In addition, they demonstrate the ability of mathematical modeling to analyze nonlinearity and inhomogeneity within these systems.

Keywords: Synthetic biology; genetic regulatory networks; complex dynamics; mathematical modeling; repressilator; artificial cell differentiation; synchronization; noise; cellular decision making.

PACS numbers: 05.45.Xt, 05.65.+b, 87.10.Tf, 87.16.Yc, 87.18.cf, 87.18.Gh

\section{Introduction}

Synthetic biology is a developing field which utilizes biology, mathematics, computation and engineering to construct synthetic genetic networks using engineering principles. A genetic network consists of a number of genes and additional molecular parts such as promoters and operators, where protein production is regulated through nonlinear positive and/or inhibitive feedback. Synthetic genetic networks serve two purposes; as stripped-down networks which mimic existing complex natural pathways or as nanorobots which perform controlled, predictable functions.

Since its inception in 2000, with the development of two fundamental simple networks, the toggle switch ${ }^{1}$ and the repressilator, ${ }^{2}$ there have been a vast number of proof-of-principle synthetic networks developed and modeled. These include transcriptional, metabolic, coupled and synchronized oscillators, ${ }^{3-7}$ networks with both oscillator and toggle switch functionality, ${ }^{8}$ calculators,${ }^{9}$ pattern formation inducers, ${ }^{10}$ learning systems ${ }^{11}$ optogenetic devices, ${ }^{12-14}$ and logic gates and memory circuits, ${ }^{15-21}$ to name but a few.

As further developments are made towards the construction of robust and predictable genetic networks, it becomes clear that synthetic genetic networks have the potential to affect and effect a lot of applications in the biomedical, therapeutic, diagnostic, bioremediation, energy-generation and industrial fields. ${ }^{22-25}$ This will be enabled when simple synthetic networks start to be assembled, coupled together and with natural networks ${ }^{18}$ in increasingly large and complex structures, in the same way that complex electrical circuits are put together from simple and basic electrical components. ${ }^{22,26}$ In line with engineering principles, this calls for 
robust, programmable, standard and predictable assemblies which avoid collateral cross-talk with cellular parts.

As more elaborate synthetic networks are developed, however, special attention needs to be given to how the individual components function together as a whole. In nonlinear and nonequilibrium systems of coupled components or components within stochastic settings, categories under which synthetic genetic networks fall, complex, interesting and unexpected dynamics can be observed. ${ }^{27}$ This includes entrainment, ${ }^{28,29}$ cell differentiation ${ }^{30}$ and coherence resonance. ${ }^{31,32}$

Unintuitive dynamics arise due to the interactions of the individual components which cause qualitative changes in system parameters, which can in turn lead to spontaneous self-organization, coordination and synchronization of dynamics. ${ }^{33}$ This is a part of the phenomenon of emergent properties in complex systems, wherein dynamics are observed in the assembled system, which were not observed within the individual parts, even though it is these individual parts themselves which are leading to complex interactions and behavior. In addition to these complex structured assemblies being nonlinear, they are often inhomogeneous and in nonequilibrium due to the effects of intra- and extra-cellular stochasticity. ${ }^{34}$ This can have a constructive effect, unexpectedly leading to noise-induced and noisesustained spatiotemporal ordering, phase transition and organization. ${ }^{34,35}$

In this review, four different studies looking at cases of complex and unexpected dynamics within coupled and stochastic simple synthetic networks through a mathematical and analytical lens will be discussed. These connect to diverse natural phenomena observed in a wide range of biological organisms including humans, ${ }^{40-42}$ animals, ${ }^{43}$ plants $^{44}$ and bacteria. ${ }^{45}$ The simple networks discussed here act as rudimentary representative networks of more complex natural counterparts. The synthetic models used are small and large populations of the repressilator ${ }^{2}$ with phase-repulsive and phase-attractive quorum-sensing, ${ }^{45}$ the one-gene Goodwin oscillator $^{46}$ within a stochastic environment and small and large toggle switches ${ }^{1}$ within a stochastic environment. These networks are used to model systems such as the circadian rhythm in chronobiology ${ }^{47}$ and cellular decision making which drives differentiation, pattern formation and apoptosis. ${ }^{49,50}$

The phenomena focused on are synchronization, clustering, inhomogeneity and chaos kinetics in coupled repressilators and how these are affected by the size of the system under consideration, the role of noise in regulating coherence and synchronization in a population of Goodwin oscillators and speed dependant cellular decision making in bistable systems, wherein an incoming signals' acceleration towards steady state can bias the systems' stability. In all four cases, the review begins with an explanation of the system and the mathematical model used to analyze it. The methods and techniques used such as bifurcation analysis, direct numerical calculations, Lyapunov theory and mean field theory are touched upon briefly. Throughout the review, we will refer to potential advantages and applications within natural genetic networks, such as adaptability and cell differentiation. 


\section{Clustering, Oscillation Death, Chaos and Inhomogeneity in Genetic Networks with Phase Repulsive Quorum Sensing}

Natural genetic networks do not usually operate in isolation. In both bacterial and eukaryote organisms, cells communicate amongst each other. In this section we review cellular behavior related to cell-to-cell communication with a focus on synchronization and desynchronization effects of autonomously oscillating and coupled genetic networks. We concentrate on the repressilator as an example of a synthetic genetic oscillator network and a prototype model of a circadian oscillator. In addition, we consider two different types of coupling, namely a phase-attractive and a phase-repulsive coupling, which can lead to multistability, oscillation death, chaotic dynamics and noise induced effects among others, as will be seen below.

A common method of communication between bacteria, which will be utilized here in conjunction with the repressilator, is quorum sensing. It is based on the relatively free diffusion of small molecules, known as autoinducers (AIs), through the bacterial membrane. In this way, bacteria are able to sense and respond to the local density of similar cells around them by monitoring the level of expression of AI-controlled genes. ${ }^{45}$ In order to understand how such collective phenomena emerge from passive intercellular communication, we make use of synthetic approaches, including mathematical modeling. The Lux system described below has been used, for instance, as a communication module to build a synthetic mechanism for programmed population control in bacterial populations. ${ }^{56}$

Quorum sensing in bacteria shares common features with the community effect of differentiating mammalian cells. The precursor cells generated by an embryonic induction tend to stay together and form a cell group of like character, despite the fact that these cells proliferate and their surrounding environment changes as a consequence of morphogenesis. Cell-to-cell communication by a diffusible factor also plays a crucial role in this case. ${ }^{40,41}$ Links between the two distinct classes of species and mechanisms may support the vice versa understanding of natural and synthetic genetic networks.

\subsection{The repressilator with quorum sensing coupling}

The repressilator is a common motif in natural genetic networks ${ }^{51-53}$ and is used as a prototype of a synthetic genetic clock built by three genes, where the protein product of each gene represses the expression of another in a cyclic manner. ${ }^{2}$ When constructed experimentally, the network expressed near-harmonic oscillations in protein levels. In this design, ${ }^{2}$ the gene lacI expresses protein LacI, which inhibits transcription of the gene tet $R$. The product of the latter, TetR, inhibits transcription of the gene $c I$. Finally, the protein product CI of the gene $c I$ inhibits expression of lacI and completes the cycle [see left-hand module in Fig. 1(a)].

Cell-to-cell communication was incorporated to the repressilator design by adding an additional feedback loop to the network scheme that is based on the Lux quorum sensing mechanism. ${ }^{54}$ The genetic module, which can be placed on a 


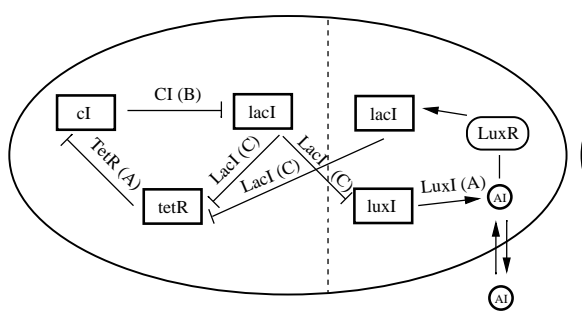

(a)

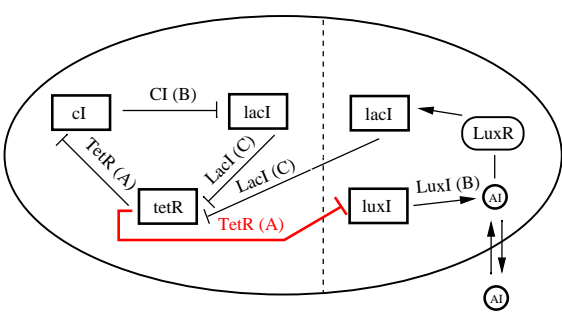

(b)

Fig. 1. Scheme of the repressilator (left module) plus quorum sensing (right module) governing cell-to-cell communication with (a) reinforcing coupling, (b) repressive coupling. The repressilator consists of three genes tetR, $c I$ and $l a c I$ which produce proteins TetR, CI and LacI respectively, represented by $A, B$ and $C$ in the mathematical model. The quorum sensing module consists of the luxI gene, the LuxR protein and AI inducer molecules which can transfer between cellular membranes. Reactions between the components are either excitatory (arrow-head lines) or repressive (blunt-head lines).

separate plasmid, involves two other proteins; ${ }^{54-56}$ LuxI, which produces a small AI molecule that can diffuse through the cell membrane, and LuxR, which responds to the AI by activating transcription of a second copy of the gene lacI. The additional quorum sensing feedback loop can be connected to the basic repressilator in such a way that it reinforces the oscillations of the repressilator [Fig. 1(a)] or competes with the overall negative feedback of the repressilator [Fig. 1(b)]. The first one leads to phase attractive coupling for robust synchronized oscillations, ${ }^{54}$ whereas the latter one evokes phase-repulsive influence, ${ }^{57-59}$ which is the key to multi-stability and rich dynamics including chaotic oscillations. ${ }^{60-62}$ Thus, through a single rewiring in the connection between the basic repressilator and the additional quorum sensing feedback loop, the entire dynamics of the cellular population are significantly altered. As a consequence, the previously favored in-phase regime becomes unstable.

The oscillator population will contain stochastic differences from cell to cell, ${ }^{2}$ giving rise to a broad distribution in the frequencies of the individual clocks which can impair the expression of a coordinated response of the cell ensemble. However, phase attractive coupling ${ }^{54}$ counteracts the desynchronization of the orchestrated behavior. As the cell density increases, diffusion of extracellular AI molecules into neighboring cells provides a mechanism of intercell coupling, which leads to a partial frequency locking of the cells. Finally, when the cell density is large enough, perfect locking and synchronized oscillations are observed through a transition via clustering. In this case, the system of cells behaves as a macroscopic clock with a well-defined period. This result indicates that a transition from an unsynchronized to a synchronized regime develops as the strength of coupling increases (due to an increase in cell density). This behavior is robust in the presence of noise. ${ }^{54}$

On the other hand, placing the luxI gene under inhibitory control of the repressilator protein TetR [Fig. 1(b)] will lead to repressive and phase-repulsive coupling 


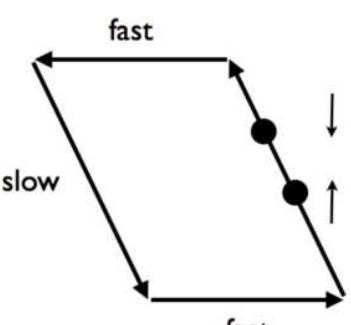

fast

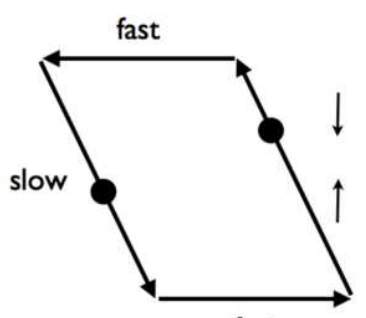

fast

Fig. 2. Principle scheme of phase-repulsive coupling realized by coupling via the slow variable in a system with two variables with different time scales, slow (horizontal direction) and fast (vertical direction). The coupling is in the slow direction and tries to pull the coupled oscillators, represented by black dots, together in the direction of the arrows.

which induces more complex dynamics. This phenomenon is common in several biological systems, including neural activity in the respiratory system, ${ }^{42}$ the brain of songbirds, ${ }^{43}$ the jamming avoidance response in electrical fish, ${ }^{63}$ the morphogenesis in Hydra regeneration and animal coat pattern formation. ${ }^{64}$ Phase-repulsive coupling arises from diffusive coupling via a slow variable and a weak time scale separation in slow and fast variables, caused by different protein life-times (Fig. 2).

In particular, we consider a population of repressilators with identical parameters, with phase-repulsive coupling [Fig. 1(b)] through fast diffusion of the AI molecule across cell membranes. The mRNA dynamics are described by the following Hill-type kinetics with Hill coefficient $n$ :

$$
\begin{aligned}
& \dot{a}_{i}=-a_{i}+\frac{\alpha}{1+C_{i}^{n}}, \\
& \dot{b}_{i}=-b_{i}+\frac{\alpha}{1+A_{i}^{n}}, \\
& \dot{c}_{i}=-c_{i}+\frac{\alpha}{1+B_{i}^{n}}+\frac{\kappa S_{i}}{1+S_{i}},
\end{aligned}
$$

where the subindex $i$ specifies cell $i . a_{i}, b_{i}$ and $c_{i}$ represent the concentrations of mRNA molecules transcribed from the tetR, $c I$ and lacI genes, respectively. The parameter $\alpha$ is the dimensionless transcription rate in the absence of a repressor. The parameter $\kappa$ is the maximum transcription rate of the LuxR promoter.

The protein dynamics are given by

$$
\begin{aligned}
& \dot{A}_{i}=\beta_{a}\left(a_{i}-A_{i}\right), \\
& \dot{B}_{i}=\beta_{b}\left(b_{i}-B_{i}\right), \\
& \dot{C}_{i}=\beta_{c}\left(c_{i}-C_{i}\right),
\end{aligned}
$$

where variables $A_{i}, B_{i}$ and $C_{i}$ denote the concentration of the proteins TetR, CI and LacI, respectively. The dynamics of the proteins are linked to the amount of responsible mRNA, and the parameter $\beta_{a, b, c}$ describes the ratio between mRNA and 
the protein lifetimes (inverse degradation rates). The model is made dimensionless by measuring time in units of the mRNA lifetime (assumed equal for all genes) and the mRNA and protein levels in units of their Michaelis constant. The mRNA concentrations are additionally rescaled by the ratio of their protein degradation and translation rates. ${ }^{54}$

The third term on the right-hand side of Eq. (3) represents activated production of lacI by the AI molecule, with concentration inside cell $i$ denoted by $S_{i}$. The dynamics of CI and LuxI can be considered identical, given that their production is controlled by the same protein (TetR). Hence, lifetimes of the two proteins are assumed to be equal and the synthesis of AI $S_{i}$, can be considered to be expressed at a rate depending on the concentration $B_{i}$ of the protein CI. Taking also into account the intracellular degradation of the AI and its diffusion toward or from the intercellular space, the dynamics of $S_{i}$ are given by:

$$
\dot{S}_{i}=-k_{s 0} S_{i}+k_{s 1} B_{i}-\eta\left(S_{i}-S_{e}\right),
$$

where the diffusion coefficient $\eta$ depends on the permeability of the membrane. Because of the fast diffusion of the extracellular AI molecules $\left(S_{e}\right)$ compared to the repressilator period, we can apply the quasi-steady-state approximation to the dynamics of the external $\mathrm{AI}^{54}$ which leads to

$$
S_{e}=Q \bar{S} \equiv Q \frac{1}{N} \sum_{i=1}^{N} S_{i} .
$$

The parameter $Q$ is the coupling coefficient, defined as:

$$
Q=\frac{\left(\delta N / V_{\text {ext }}\right)}{\left(k_{s e}+\delta N / V_{\text {ext }}\right)},
$$

where $N$ is the number of cells (repressilators), $V_{\text {ext }}$ is the total extracellular volume, $k_{s e}$ is the extracellular AI degradation rate, and $\delta$ is the product of the membrane permeability and the surface area.

The coupling coefficient $Q \in(0,1)$ is proportional to the cell density and can be controlled in a chemostat experiment by changing the total culture volume. Therefore, in the numerical investigations that follow we use $Q$ as a free and experimentally tunable parameter to study the behavior of the cell ensemble under different environmental conditions. The other model parameters cannot be changed experimentally in a simple way and so we keep these parameters fixed and dimensionless throughout the investigations. See Refs. 60, 61 and 62 for details. All parameters used are biologically reasonable.

\subsection{The dynamical regimes for a minimal system of repressilators coupled via phase-repulsive quorum sensing}

The effect of coupling on the dynamics of intercell genetic networks can be understood by investigating a minimal system of two oscillators with phase repulsive 


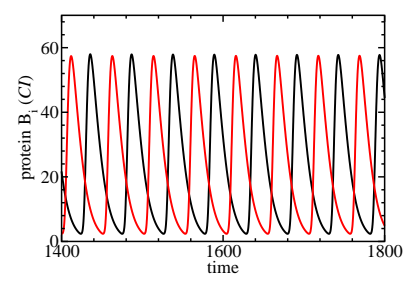

(a)

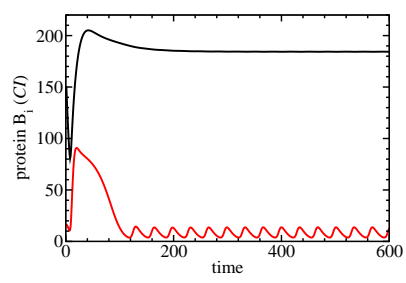

(b)

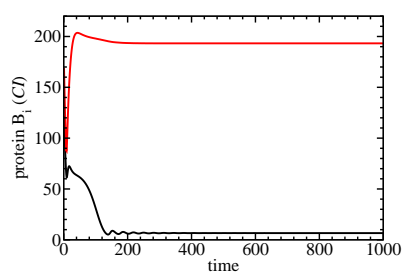

(c)

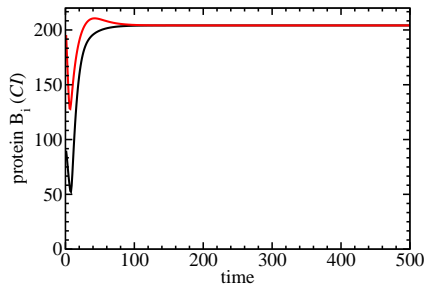

(d)

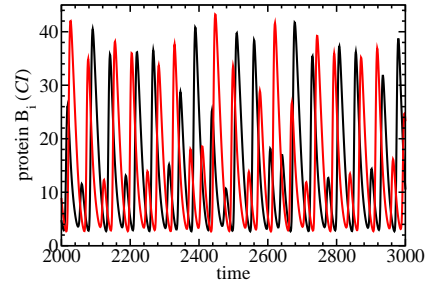

(e)

Fig. 3. (Color online) Typical time series of the CI protein concentration in a cell for the five stable regimes in the smallest ensemble of $N=2$ coupled repressilators for (a) $Q=0.1$ oscillations, (b) $Q=0.3$ inhomogeneous limit cycle (IHLC), (c) $Q=0.4$ inhomogeneous steady state (IHSS), (d) $Q=0.4$ homogeneous steady state (HSS), and (e) $Q=0.8$ chaotic oscillations.

coupling. Figure 3 shows representative time traces, obtained by direct numerical calculations of a population of $N=2$ coupled repressilators, for increasing coupling strength $Q$. The different dynamical regimes found are self-sustained oscillatory solutions [Fig. 3(a)], inhomogeneous limit cycles (IHLC) [Fig. 3(b)], inhomogeneous steady states (IHSS) [Fig. 3(c)], homogeneous steady states (HSS) [Fig. 3(d)] and chaotic oscillations [Fig. 3(e)], all of which exist for biologically realistic parameter ranges.

Both inhomogeneous solutions (IHLC and IHSS) are interesting, in that they show different behavior of isogenetic cells in the same environment. One cell maintains a high level of protein CI whereas the other cell keeps a low CI concentration, which in turn implies a high LacI concentration. Both cells are able to specialize as a LacI or CI producer and only the history (initial condition in the numerical experiments) determines the CI state. Both inhomogeneous states are combined states and differ from a bistable system in that each protein level cannot be occupied independently.

\subsubsection{Stability and attractors of the regimes}

In order to analyze the dynamical regimes, the coupling parameter $Q$ is varied. We make use of two methods which complement each other; bifurcation analysis and direct numerical calculations. See Refs. 61,62 and 65 for more details of the bifurcation analysis. 
All regular stable dynamical regimes found with direct numerical calculations [Figs. 3(a)-(d)] can also be seen in the bifurcation diagram [Fig. 4(a)]. To compare the bifurcation analysis with the results of direct calculations, we calculated 1000 time series for the system of two coupled repressilators with different random initial conditions. These cover the 14-dimensional phase-space of the system (seven degrees of freedom per oscillator) densely enough such that one can detect stable coexisting attractors with a basin of attraction. Several dynamical regimes coexist at the same $Q$ value and form the multi-stability of the system. For example, for low cell densities when $Q<0.129$, anti-phase self-oscillations are the only stable regime but at $Q=0.129$, the HSS (black) stabilizes through a limit point bifurcation, and coexists with the oscillatory solution (yellow).

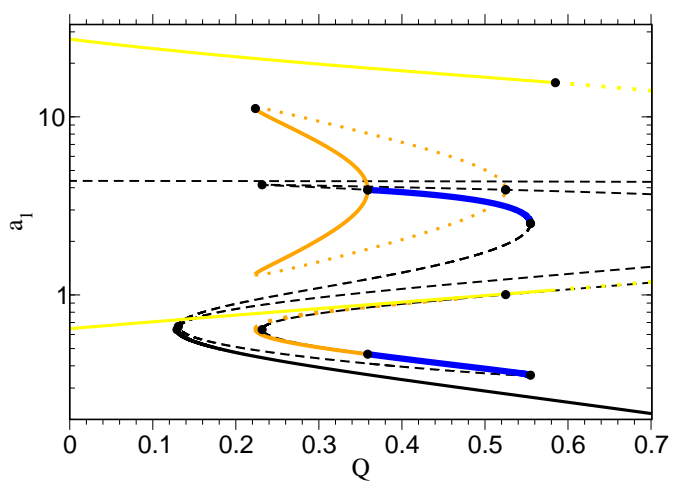

(a)

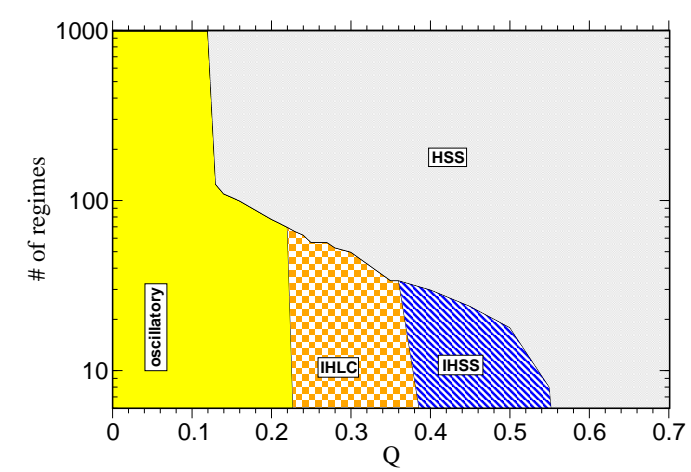

(b)

Fig. 4. (Color online) For $N=2$ repressilators, comparison between (a) bifurcation analysis showing variable $a_{1}$ (tetR mRNA) versus coupling $Q$ and (b) direct calculations with random initial conditions showing the number of regime solutions versus coupling $Q$. Note the logarithmic scale of both ordinates in the two plots. The oscillatory regime's maximum and minimum values are represented by a yellow solid line (a) and a yellow area (b); the IHLC by solid orange lines (a) and an orange-white chess board pattern (b); the IHSS by solid blue lines (a) and a small blue striped area (b); the HSS by a solid black line (a) and a grey area (b). 


\section{Y. Borg et al.}

Results show that the direct numerical calculations and the bifurcation analysis coincide in the onset of the HSS, the IHLC, the IHSS and the mutual exclusion of IHLC and IHSS. Only the existence of stable oscillations for $Q>0.2236$ could not be seen by the direct numerical method, revealing the dominance of the singlefixed-point solution; at $Q=0.3,50$ solutions end up in the IHLC, while all other 950 are in the HSS and none are in the oscillatory regime [Fig 4(b)].

The chaotic dynamics in Fig. 3(e) can be seen as an additional source of uncertainty beside the known intrinsic and extrinsic noise sources in genetic networks. ${ }^{66,67}$ The bifurcation analysis [Fig. 4(a)] predicts unstable anti-phase oscillations beyond a torus bifurcation for $Q \approx 0.587$, at which stable oscillations disappear and the CI protein trajectory fills up a growing dense space [Fig. 5(b)]. To find the stable solutions, one can perform direct simulations for different coupling $Q$ values. For $Q<0.67$, the resulting self-oscillations are stable and resistant to small

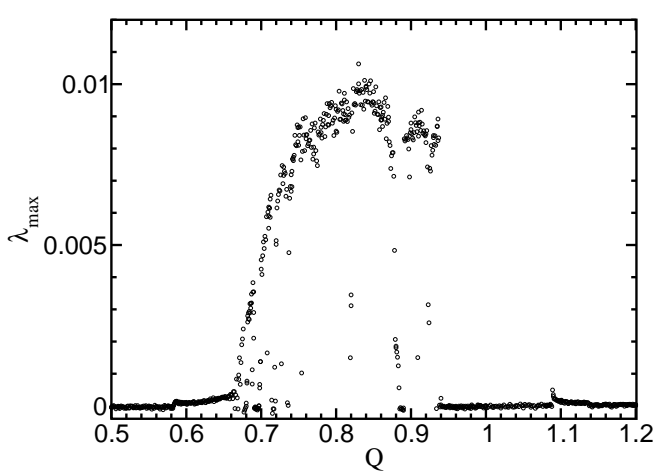

(a)

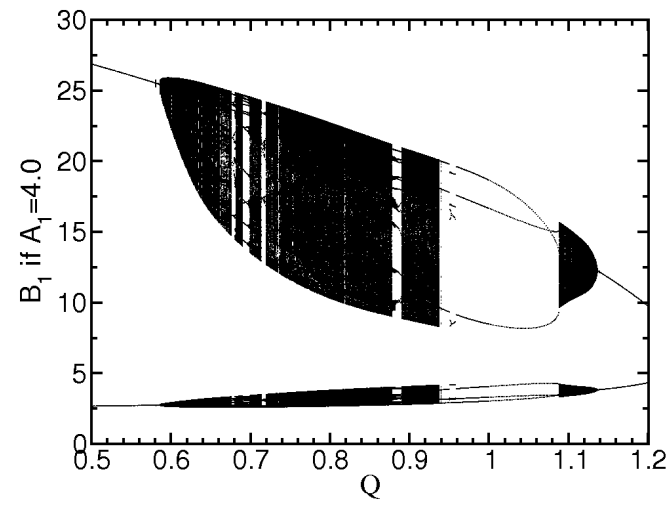

(b)

Fig. 5. For $N=2$ repressilators, (a) the maximal Lyapunov exponent $\lambda_{\max }$ versus coupling $Q$, (b) the corresponding bifurcation diagram computed as a series of Poincaré sections, with the ordinate showing the value of the $B_{1}(\mathrm{CI})$ protein versus coupling $Q$. 
perturbations in the initial conditions and dynamical noise. The maximal Lyapunov exponent remains unaffected by the torus bifurcation at $\lambda_{\max } \approx 0$ [Fig. 5(a)]. ${ }^{68}$

At $Q \approx 0.67$ the torus attractor becomes unstable and $\lambda_{\max }$ increases significantly above zero, marking the onset of chaotic behavior. The chaotic regime ranges up to $Q \approx 0.937$ and is interrupted by periodic windows at some intermediate ranges of $Q$. The chaotic region ends abruptly at $Q \approx 0.937$, at which point $\lambda_{\max }$ declines to zero and the bifurcation diagram reveals a relatively simple structure. Values of $Q$ beyond 1 are outside the biologically relevant range but important from the dynamical system's viewpoint. It is interesting to note that parameter estimation methods for chaos in genetic networks have now been developed. ${ }^{69}$

Both regular and chaotic self-oscillatory regimes have a rather small basin of attraction, which makes it very difficult to reach them from a random sampling of initial conditions in the phase-space [Fig. 4(b)]. Thus, it is not clear whether chaotic dynamics would be observed in an experimental implementation of our model. However, one can envisage an experimental protocol in which a cell population starts from the self-oscillatory regime with small $Q$, i.e., small cell density, and smoothly increases its density due to replication. In that case, one can expect the system to end in a self-oscillatory regime at high cell density $Q$, and eventually in a chaotic regime. A "hidden" stable chaotic attractor with a small basin of attraction can be important for noisy systems. ${ }^{70}$ Noise can take the dynamics of the genetic network from a regular or close-to-regular behavior (smooth oscillations, HSS, IHSS or IHLC) to the basin of the chaotic attractor, leading to the excitation of unstable periodic orbits with erratic and sudden responses. This can be beneficial for the genetic network since it increases the flexibility and speed of state changes (adaptation).

\section{Systems Size Effects in Coupled Genetic Networks}

Bacterial colonies typically consist of a population of cells and hence we extend the results to large ensembles of represillators with phase-repulsive quorum sensing. Figure 6(a) plots the frequency of stable regimes for increasing $Q$ for an ensemble of $N=100$ coupled identical cells obtained from direct calculations with random initial conditions. The four main regimes HSS, IHSS, IHLC and regular self-oscillations observed in the minimal system can be detected in a large system.

The results [Fig. 6(a)] reveal a transition from self-oscillations to a single stable fixed point as the coupling $Q$ increases. This transition is gradual, and exhibits a multiplicity of regimes similar to the previous situation for $N=2$ [Fig. 4(b)]. For $Q \lesssim 0.13$ only self-oscillations are found (Fig. 9). At $Q \approx 0.13$ the basin of the self-oscillatory regime disappears, and IHLC arise [Figs. 7(c) and 8], such that there is no mixing of the two populations. As in the minimal case of $N=2$, the basin of the IHLC coexists with the basin of the HSS [Fig. 6(a)]. This single fixed point attractor becomes more likely for larger coupling strengths $Q$. In the range $Q \in[0.2,0.23]$, a second transition takes place, through which the IHLC disappears 


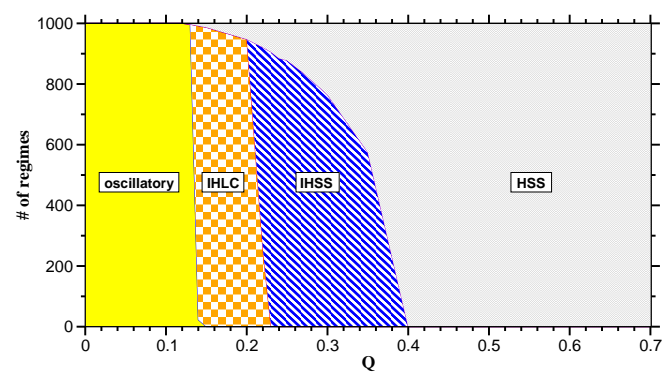

(a)

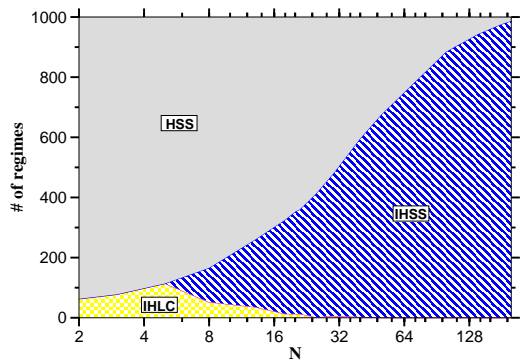

(b)

Fig. 6. (Color online) Direct calculations showing distribution of (a) the stable regimes for increasing coupling strength $Q$ for a large ensemble of $N=100$ repressilators, (b) the effect of the repressilator population size $N$ on the distribution of stable regimes for a particular coupling strength $Q=0.24$.

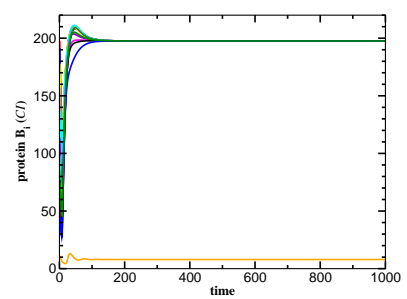

(a)

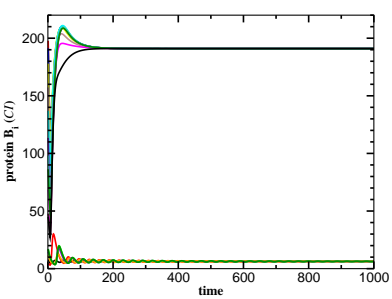

(b)

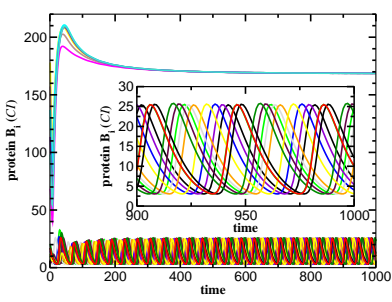

(c)

Fig. 7. Time series of CI protein in a fixed system size of $N=18$ repressilators and coupling $Q=0.3$ showing the IHSS in (a) 17:1 cluster distribution, (b) 12:6 cluster distribution, (c) an example of IHLC coexisting for the same parameters. The IHLC example contains six oscillators in the upper CI state and 12 in the lower one. The three plots illustrate the coexistence of IHLC and IHSS for the same coupling $Q$.

and the IHSS regime, corresponding to fully developed oscillation death, arises. In this regime, all cells differentiate into two different clusters at high and low CI levels in several different ratios [Figs. 7(a) and 7(b)]. Since each cluster represents the production of a different protein, this regime can be interpreted as a mechanism of artificial differentiation in an isogenic population of cells. The basin of the IHSS regime also coexists with the basin of the single fixed point attractor, which becomes increasingly more likely for increasing $Q$, until it turns into the dominant attractor of the system for $Q \gtrsim 0.4$. The dynamical regimes described above persist in a noisy environment. For instance, protein fluctuations larger than $25 \%$ of their mean level do not alter the clustering attractor in the multistable parameter range.

Compared to smaller system sizes, the inhomogeneous states are more likely to occur, at the expense of the HSS [Figs. 4(b) and 6(b)]. Figure 6(b) shows the growing basin of attraction of the inhomogeneous solutions when the system size grows. The likelihood of the IHSS becomes the dominant regime for cell colonies with $N \gtrsim 32$ cells. This is similar to the differentiation mechanism and community effect of stem 
cells. ${ }^{40,41}$ The artificial cell differentiation in the repressilator system is based on phase repulsive coupling in a weakly nonlinear system with slow-fast dynamics. Thus, the basic mechanism is extendable to other synthetic genetic oscillators and possibly inside natural genetic networks regulating cell differentiation.

\subsection{Clustering and enhanced complexity of the inhomogeneous regimes}

A small increase in the system size is sufficient to alter the balance between the coexisting regimes. This underlines the connection between the size of the population and its dynamical behavior. It is well known that a large population of genetically identical cells may exhibit diverse phenotypic states even under almost identical environmental conditions. This heterogeneity is manifested by the existence of several subgroups or clusters. Clustering can be defined as a stable dynamical state characterized by the coexistence of several subgroups where the oscillators exhibit organized identical or nearly identical behavior. Clustering is a well known property, especially for globally coupled systems, and has been investigated in identical phase, ${ }^{71,72}$ salt-water ${ }^{73}$ and electrochemical oscillators, ${ }^{74,75}$ in synthetic genetic networks ${ }^{42}$ and in populations of chaotic oscillators, ${ }^{76-78}$ among other cases.

The direct numerical calculations' analysis (Fig. 6) revealed a significant enlargement $(\approx 50 \%)$ of the IHSS stability interval in comparison to the minimal case of $N=2$ coupled oscillators (for details see Ref. 61 ). This is a result of clustering, or more specifically, of the increased number of possible distributions of the oscillators between the two stable protein levels. Each cluster distribution (respective separation of cell in a high or low CI state) forms an individual solution (stable or unstable) in the bifurcation analysis, which leads to a very complex structure of bifurcation diagrams for systems with $N>2$. The different stable cluster distributions are located on separate branches of the bifurcation continuation.

We focus on the main differences from the minimal case $N=2$ and discuss their relevance to genetic networks. Figures $7(\mathrm{a})$ and $7(\mathrm{~b})$ show time traces for two different cluster decompositions in the IHSS regime for a system of $N=18$. Each possible decomposition shows slightly different stable protein concentration levels, and hence tuning of the protein levels can be accomplished by choosing a specific $Q$ interval. This specific effect enhances, on the one hand, the biotechnological applications of synthetic genetic networks by providing a possible method for fine manipulation of the protein concentration level, and on the other hand can be seen in natural networks as typical adaptability of a cell population to environmental conditions.

The population displays even more complicated behavior when analyzing the clustering effect in the IHLC regime. This complexity is manifested through the formation of sub-clusters in the lower (oscillatory) state, where oscillators exhibit identical behavior within a single sub-cluster, but with various phase relations among them (Fig. 8). Moreover, the ratio of the oscillators distributed in the upper versus 


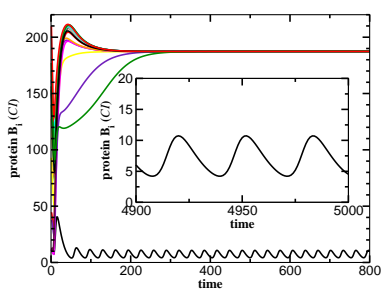

(a)

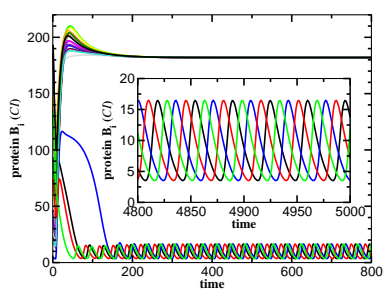

(d)

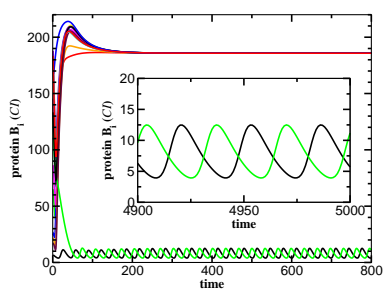

(b)

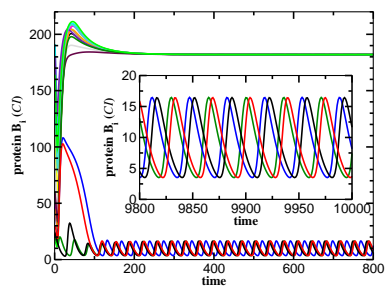

(e)

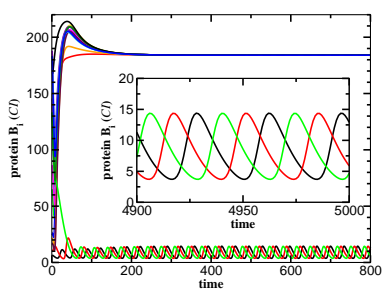

(c)

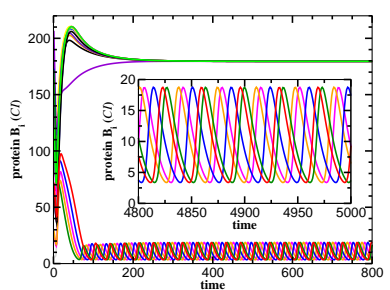

(f)

Fig. 8. Examples of time-series showing CI protein concentrations for different IHLC distributions and phase relation in the sub-threshold oscillations in an ensemble of $N=18$ repressilators for the same coupling $Q=0.2$.

lower CI protein affects the amplitude and period of the limit cycle oscillations located in the lower protein level. In the shown example for $N=18$ and $Q=0.2$ (Fig. 8) the period varies from $\approx 31.7$ for the distribution of one oscillator in the low CI state and all other 17 in the high state [Fig. 8(a)] up to $\approx 36.5$ for the distribution of five oscillators in the low and 13 in the high CI state [Fig. 8(f)]. In the discussed case every additional oscillator in the lower CI level of the IHLC lengthens the period by $\approx 1.2$ time units, which leads to a significant change in the period between different distributions.

\subsection{Clustering due to regular oscillations in cell colonies}

Similarly to the minimal system of $N=2$, the repressilator colony can express two main types of oscillator behavior depending on the coupling strength $Q$ : regular oscillations with stable cluster formation and chaotic self-oscillations with temporary cluster formation. We will denote this as grouping in the following discussion. In order to distinguish between separate cluster formations for increasing population sizes via numerical simulations, we use the following definition: oscillators $i$ and $j$ belong to the same cluster $K$ at time $t$ if the difference between the internal AI concentrations $S_{i}(t)$ and $S_{j}(t)$ is smaller than a pre-defined value $\varepsilon=0.001$. Using this criteria, the resulting cluster plots classify the temporal grouping of the oscillators [Figs. 9(b), 10, 11(d) and 12], highlighting the difference in the protein concentrations of separate cells over time. 


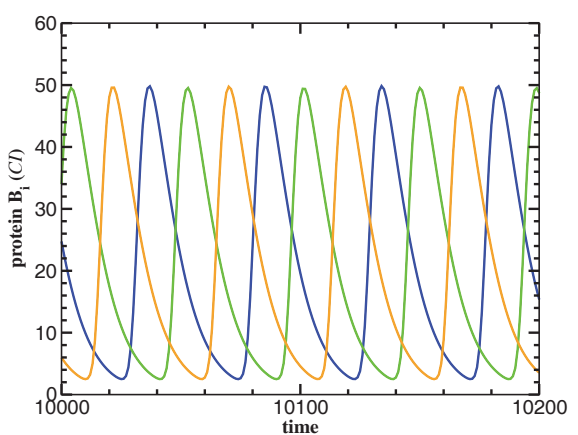

(a)

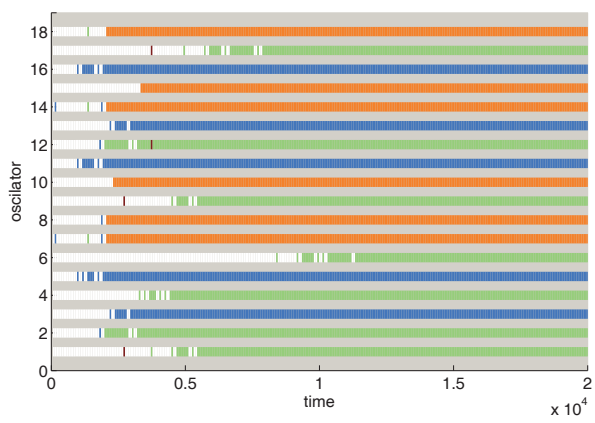

(b)

Fig. 9. Three-cluster decomposition for a system of $N=18$ repressilators. (a) Time series of the CI protein concentration in the regular oscillating regime at coupling $Q=0.3$, exhibiting three-cluster decompositions with $7: 6: 5$ distribution of cells and a phase shift of about $\sim \frac{2 \pi}{3}$ among them. After a transient of about $1.2 \times 10^{4}$ time units, a synchronous behavior inside each cluster emerges and the individual dynamics of the cells inside each cluster are indistinguishable, (b) Cluster-plot representation of the time-series in (a). White clusters represent a free-running oscillator, which does not belong to any of the clusters. The coloring in the time series corresponds to the colouring in the cluster plot.

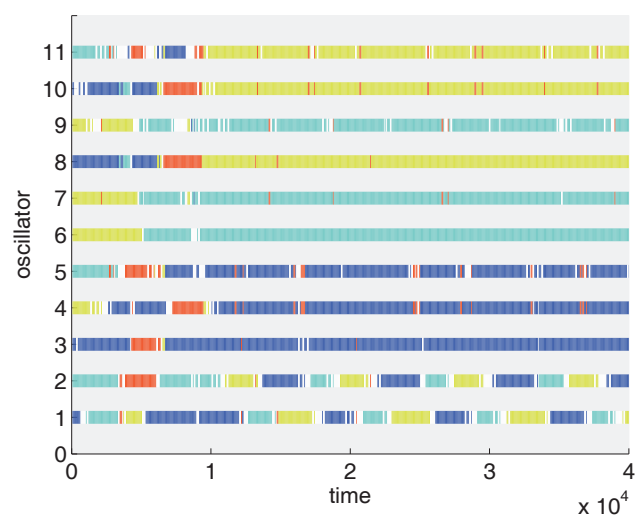

Fig. 10. Cluster plot of $N=11$ nonidentical repressilators in the self-oscillatory regime at coupling $Q=0.5$ as the parameter $\alpha_{i}$, the dimensionless transcription rate in the absence of a repressor, is varied in each repressilator. $\alpha_{i}$ increases from bottom to top.

In systems where $N>5$, clustering is observed in the regular oscillatory regime. After a transient, the onset of clustering occurs [Fig. 9(b)]. ${ }^{30,71,79}$ The three-cluster decomposition dominates, with a nearly equal number of oscillators in each one, and a distinct phase relation between separate clusters (for details see Table 2 in Ref. 61). This phenomenon dominates for large system sizes, over wide ranges of coupling, $Q$.

Time series of the separate clusters in a regular oscillating regime (the oscillators within each cluster display synchronous behavior) are given in Fig. 9(a). The long 


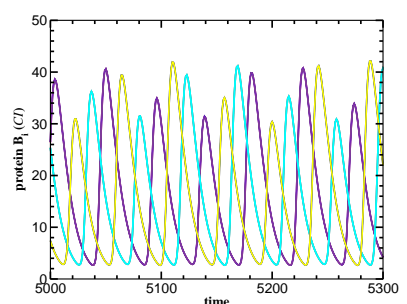

(a)

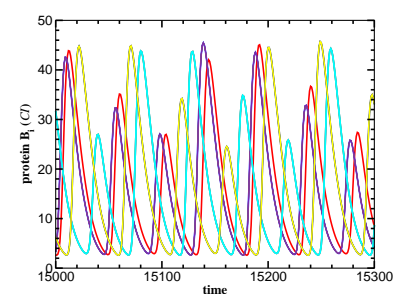

(b)

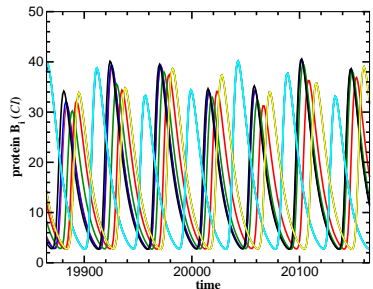

(c)

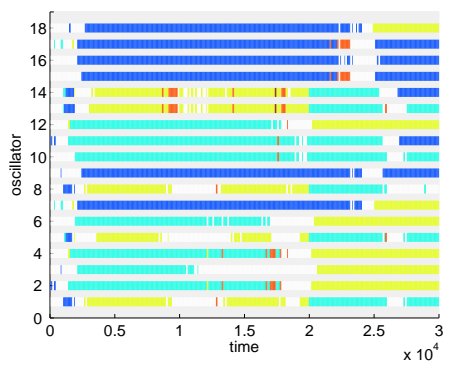

(d)

Fig. 11. Chaotic behavior seen in (a), (b), (c) time series of the same system of repressilators taken at different time-points and (d) the corresponding cluster plot over the entire time span $\left(0,3 \times 10^{4}\right)$ in the self-oscillatory regime of $N=18$ oscillators with weak chaotic behavior and long lasting grouping at $Q=0.6$. The color coding between the four figures matches.

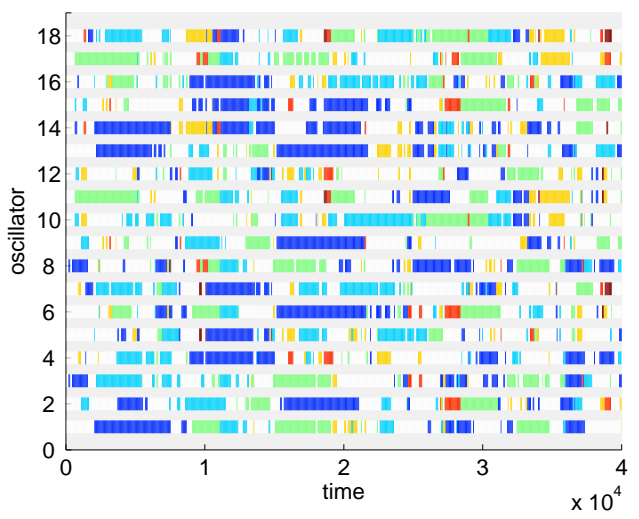

Fig. 12. Cluster plot in the self-oscillatory regime for $N=18$ repressilators with strong chaotic dynamics and short-lived grouping at coupling $Q=0.75$.

transient in the simulation looks unphysiological at first glance, but all simulations are drawn from random initial conditions with a very large diversity amongst the cells. We use these unrealistic initial conditions in order to underline the ability of the system to form stable clusters under any condition. After proliferation, the 
daughter cells are in a similar phase as the mother, which decreases the time to form stable clusters significantly. Normally, stronger coupling lengthens the period of coupled systems, ${ }^{29,80}$ but the situation is different in the present case because $Q$ controls the re-influx of AI and a higher internal AI concentration shortens the repressilator cycle. Compared to the coupling strength $Q$, the system size $N$ and the cluster composition have a minor influence on the period. The separation into three clusters can provide the population of cells with high stress resistance, because at any given time the cells in the different clusters are in different states of the limit cycle, and hence each cluster will be affected differently by sudden environmental stresses such as chemicals or lack of nutrients.

\subsection{Parameter heterogeneity on the regular-attractor regime}

The assumption that the elements of the system are identical and deterministic (differing only in the initial conditions) is very strong, since cellular populations are heterogenous. It is important to account for diversity among parameter values in separate cells by introducing, for example, mismatch in the $\alpha$ parameter values. Introducing diversity in $\alpha$ is realistic since this parameter defines the expression strength of genes within the repressilator, which is proportional to the concentration of repressilator plasmids present in the cell. In particular, we consider here a system of size $N=11$, where for each cell $i=1, \ldots, 11$ different $\alpha$ 's are assigned from a defined set of values. This leads to a variability larger than $3 \%$ in the oscillation periods. The control of the number of plasmid copies in experiments was discussed in Ref. 81 and can be coordinated with the cell's growth and division.

In the presence of diversity, the three-cluster decomposition remains the dominant state in the system (Fig. 10). However, in contrast to the case of identical oscillators, the two oscillators with the smallest parameter $\alpha$, i.e., the cells with the shortest period, are not phase locked and jump periodically from one of the three stable clusters to the other. Whereas identical oscillators do not have local grouping preferences due to the symmetry of the system, the heterogeneity introduced via the parameter mismatch breaks the symmetry and leads to a situation where oscillators with similar properties (i.e., similar $\alpha_{i}$ ) group together in a cluster. One could speculate that the grouping of cells with similar behavior in an oscillating cluster could be used for cell sorting and optimization of synthetic genetic circuits.

\subsection{Irregular and chaotic self-oscillations in colonies of identical cells}

As in the minimal system with $N=2$ repressilators, regular oscillations convert to irregular oscillations for high cell densities, i.e. coupling $Q$. Irregular oscillations look very similar to chaotic time series and a positive maximal Lyapunov exponent confirms the chaotic behavior for $Q>Q_{\text {chaos }} \approx 0.6$. The numerical bifurcation diagram and the development of the maximal Lyapunov exponent versus coupling 
$Q$ look similar to those of the minimal system with $N=2$ (Fig. 5). Simulation details for $N=18$ can be found in Ref. 61 . The chaotic dynamics appear gradually, with weak chaotic behavior for $Q \in[0.6,0.7]$ and fully developed chaos for $Q \in$ $[0.7,1]$.

Figure 11 shows weak chaotic dynamics of $N=18$ oscillators at $Q=0.6$, with long three- and four-grouping constellations. The cluster plot [Fig 11(d)] illustrates the interplay of long-time grouping and recurring transients with less ordered states while a rearrangement to a new grouping happens. The groupings last up to 20,000 time units, i.e., about 4000 cycles. Once the oscillators are distributed in a longliving grouping state, they oscillate synchronously within the group and cannot be distinguished by their time series until the next decomposition occurs and spreads the phases.

The second example of irregular chaotic self-oscillations (Fig. 12) illustrates a regime of fully developed chaos at high coupling $Q=0.75$. The maximal Lyapunov exponent increases significantly above zero, which confirms the chaotic character of the dynamics. Interestingly, the temporal groupings of the oscillators are conserved, but are unstable with a significantly shorter lifetime and faster mixing as compared to the weak chaotic dynamics discussed above (Fig. 11). In this typical situation (Fig. 12), the grouping of the oscillators can last up to 5,000 time units, i.e., more than 100 oscillations.

In general, it can be stated that inside the chaotic ensemble there exists a tendency to build and break temporal groups, which leads to their mixing. Many different temporal distributions of the oscillators into groups are possible, which survive over several oscillation periods until the next mixing occurs. The observed clustering and grouping effects resemble the dynamical behavior characterized for a "globally coupled map". ${ }^{82}$ The transition from an ordered to a partially ordered and turbulent phase, where the number of clusters is significantly increased is similar to the case of a weak and well-developed chaotic clustering decomposition discussed in this section. Moreover, we show that a growing system increases both the possibility for grouping formation significantly and the number of different oscillator distributions between the groups. This enhances the flexibility of the system and means that by varying environmental conditions, the population can switch between different distributions to adapt to the surroundings.

Although the chaotic dynamics observed here and the effect of intrinsic noise in synthetic oscillators ${ }^{2,3}$ have very similar manifestations despite different origins, we demonstrate that chaos is an alternative source of uncertainty in genetic networks. The chaotic dynamics and the grouping phenomena appear gradually for increasing coupling $Q$, i.e., at cell densities which can be a cause for stress. One could speculate that the population has the flexibility to respond to and survive environmental stress by distributing its cells within stable clusters. The gradual chaotic behavior enables the population to adapt the mixing velocity and the degree of diversity to the stress conditions. 
Suzuki et al. systematically investigated five gene network motifs suitable to control cell differentiation ${ }^{83}$ and found that the repressilator motif is commonly embedded in functioning networks which express chaotic dynamics under certain initial conditions and parameter sets. These findings support the idea to use the repressilator with repressive cell--cell communication as a prototype of artificial cell differentiation in synthetic biology.

\section{The Constructive Role of Noise in Genetic Networks}

Genetic networks cope with significant noisy perturbations caused internally by the inherent stochasticity of biochemical processes and externally by environmental fluctuations. ${ }^{66,67}$ Intrinsic noise is unavoidable because protein synthesis is a stochastic event, presenting a challenge for the design of any synthetic genetic network, especially small systems where many components are present at low copy numbers at or below the order of ten. On the other hand, extrinsic sources of noise arise independently of the gene network under consideration but can act on it. ${ }^{66}$ Extrinsic noise is caused by molecular species in the cell, such as RNA polymerase, cells within the population and changing environmental conditions. Noise and noise reduction strategies in genetic networks is a wide and ongoing field and we refer the interested readers to the literature e.g., Refs. 84-89. It has been shown that the interaction of noise with nonlinear systems and forces can have positive effects on the systems, such as enhanced order and regular behavior, the appearance of new behavior, improved signal response, better sensitivity and noise-induced transport. ${ }^{32,90-94}$ In the following sections, we discuss potential beneficial effects of noise in genetic networks, focusing on noise-induced oscillations and coherence.

\subsection{Noise-induced oscillations in circadian gene networks}

Biochemical clocks with intrinsic time scales can display regular oscillations in the presence of intermediate levels of stochasticity. ${ }^{95,96}$ The oscillations occur within a narrow frequency range as a temporal coherent output close to nonlinear instabilities of periodic orbits. ${ }^{97}$ The phenomena is known as coherence resonance, stochastic coherence, autonomous or self-induced stochastic resonance. Amongst these terms, we will use stochastic coherence as it describes the systems' response more appropriately. Studies as far back as $1974^{98,99}$ support the theory that under certain conditions, noise can have a constructive role and extend the range of parameters which result in oscillatory behavior. . $^{3,100,101}$

Recent experiments and mathematical models suggest that the circadian rhythm in individual cells is also a noise-induced oscillation. ${ }^{47,102,103,112}$ Circadian rhythms are autonomous 24-hour-period biochemical clocks controlled by external light which are observed in a vast range of living organisms from cyanobacteria, plants and insects to mammalian cells. The mammalian circadian clock is based on negative feedback and is hierarchically organized, mastered by the suprachiasmatic nuclei (SCN). ${ }^{113,114}$ The SCN consists of about 20,000 neurons, each equipped 
with an individual molecular clock driving neuronal activity. These clocks interact with each other, ${ }^{28,115-117}$ and their activity can be entrained by natural periodic light modulation, the day-night cycle of the sun or alternative artificial sources. Although individual cellular circadian clocks are irregular, the orchestrated rhythm of 20,000 molecular communication clocks in the SCN generate a precise and reliable central clock, which persists even in the absence of daily light rhythm under certain conditions of constant light. This will be further discussed in the following section.

In deterministic circadian clocks, mathematical models based on experimental results predict damped oscillations. It is only with the inclusion of genetic noise that the system is excited regularly and results in stochastic coherence. ${ }^{47,102,103,112}$ Hence, noise has a potentially constructive role, involved in the generation of the central rhythm which pervades the entire body from the activity pattern, to metabolism and organs. ${ }^{104-111}$

\subsection{Noise-induced synchronization and rhythms}

Under constant light conditions, SCN cells are only able to produce self-sustained oscillations when illumination levels are sufficiently low. If constant light is increased, the circadian clock undergoes a transition from a rhythmic (normal) to an arrhythmic (abnormal) behavior. ${ }^{118}$ Remarkably, the individual clocks remain in the oscillatory state but lose their synchronization, ${ }^{119}$ oscillating with different phases and eigen-frequencies due to intercell variability. The fact that increasing light leads to arrhythmicity ${ }^{119}$ suggests that illumination may have a repressive influence on the strength of coupling among clock cells. ${ }^{120}$ In this scenario, under constant darkness, the single-cell oscillators are strongly coupled and hence, are synchronized and phase-locked. The assumption of light-controlled coupling does not exclude, but rather complements, the direct effect of light on the individual cells, as it highlights the importance of synchronization for the emergence of overall circadian rhythmicity.

A hypothesis was developed to test whether random fluctuations in a constant lighting profile can sustain circadian rhythmicity in a multicellular clock, under conditions for which the clock is arrhythmic in deterministic conditions. ${ }^{121}$ The phenomenon is similar to stochastic coherence mechanisms in stochastic dynamics literature. ${ }^{93}$ It is assumed that the only relevant influence of light into the system is through the inter-oscillator coupling. The direct effect of light on the clock cells is expected to play a more important role in the case of entrainment to a lightdark cycle. In addition, it is assumed that noise affects the strength of coupling among the oscillators globally. The assumption of a chemical (rather than, for instance, an electrical) mechanism of intercell communication among SCN neurons as a synchronization factor is supported by recent experimental evidence. ${ }^{122}$

The hypothesis was validated by a mathematical model of the circadian pacemaker at the basic genetic level by using a large ensemble of globally coupled 
non-identical Goodwin oscillators. Developed over 40 years ago, ${ }^{46,51}$ the Goodwin oscillator consists of a single gene that represses itself directly or indirectly. It has been implemented in vivo using the $\mathrm{P}_{\mathrm{LlacO}-1}$ promoter. ${ }^{3,123}$ The Goodwin model ${ }^{124}$ describes circadian oscillations in single mammalian cells by means of three variables. The clock gene's mRNA $(X)$ is translated to a protein $(Y)$ which activates a transcriptional inhibitor $(Z)$ and in turn inhibits the transcription of the clock gene. The three variables form a closed negative feedback loop typical for Goodwin oscillators. Additionally, a global coupling term can be introduced ${ }^{125}$ depending on the concentration of a synchronizing factor (a neurotransmitter) in the extracellular medium. Since the SCN is a relatively small and dense area, fast dynamics of the small neurotransmitter molecules in the extracellular medium can be assumed in order to allow for the simplification of cell-to-cell communication by mean field coupling. The simplified coupling is comparable to the quorum sensing mechanism in bacterial colonies ${ }^{45}$ discussed in Sec. 2 .

The resulting model is ${ }^{121}$ :

$$
\begin{aligned}
\frac{d X_{i}}{d t} & =\nu_{x} \frac{K_{t}^{n}}{K_{t}^{n}+Z_{i}^{n}}-\delta_{x} \frac{X_{i}}{K_{x}+X_{i}}+\nu_{c} \frac{\alpha V_{i}}{K_{c}+\alpha V_{i}}, \\
\frac{d Y_{i}}{d t} & =\nu_{y} X_{i}-\delta_{y} \frac{Y_{i}}{K_{y}+Y_{i}}, \\
\frac{d Z_{i}}{d t} & =\nu_{z} Y_{i}-\delta_{z} \frac{Z_{i}}{K_{z}+Z_{i}}, \\
\frac{d V_{i}}{d t} & =\nu_{v} X_{i}-\delta_{v} \frac{V_{i}}{K_{v}+V_{i}}-\eta\left(V_{i}-Q F\right),
\end{aligned}
$$

where the index $i$ denotes different cells and $V_{i}$ represents the internal signaling molecule concentration of cell $i$. The production rates are represented by $\nu_{j}$, the degradation rates by $\delta_{j}$ and $K_{j}$ are Michaelis constants. The release of signaling molecules is assumed to be fast compared to the circadian timescale, which results in an average external signaling molecule level, represented by a mean field $F$,

$$
F=\frac{1}{N} \sum_{i=1}^{N} V_{i} .
$$

The authors distinguish between the intracellular signaling molecule concentration $V_{i}$, which directly activates expression of $X_{i}$ [Eq. (10)] and the extracellular signaling molecule concentration $F$. This coupling mechanism is similar to that suggested by García-Ojalvo et al. ${ }^{54}$ for intercell communication of synthetic gene oscillators via small AI molecules. The coupling between the individual cell oscillators is determined by the membrane permeability $\eta$ and the relative signaling molecule influx into the cell $Q$. It is assumed that light affects the signaling molecule influx, and thus $Q$ will be considered as the only parameter driving the synchronization transition through the influence of light. The diversity in the eigen-frequencies of the individual oscillators was modeled by rescaling the production and degradation 
rate constants $\left(\nu_{i}\right.$ and $\left.\delta_{i}\right)$ by a scaling factor $\tau_{i}, i=1, \ldots, N$, different for each cell. The value of these factors $\tau_{i}$ are drawn randomly from a normal distribution of mean $\bar{\tau}=1.0$ and different standard deviation $\sigma_{\tau}$.

\subsubsection{The mean field synchronization under constant light conditions}

In the absence of coupling, oscillations are uncorrelated due to different $\tau_{i}$ and initial phases. For sufficiently large ensembles, the mean response of the oscillators is mostly flat, showing arrhytmicity despite each oscillator having a clear periodic behavior. Coupling leads to synchronization amongst the circadian oscillators and rhythmic behavior in the mean field, associated with free running rhythmicity of mammalian cells under constant darkness conditions. Stronger coupling leads to a more pronounced synchronization and the amplitude of the mean field increases until full synchronization.

For the optimal expression of noise-induced rhythmicity, the system must operate close to the nonlinear bifurcation point of $Q$ corresponding to the onset of synchronization. The transition from a nonsynchronized to a synchronized state as $Q$ increases (i.e., the light decreases) was investigated with respect to the mean field. The degree of synchronization $R_{\text {syn }}$ is the ratio of the variance of the mean field of protein concentration to the mean variance of each oscillator ${ }^{54}$ and is defined as:

$$
R_{\mathrm{syn}}=\frac{\left\langle\bar{Y}^{2}\right\rangle-\langle\bar{Y}\rangle^{2}}{\frac{1}{N} \sum_{i=1}^{N}\left(\left\langle Y_{i}^{2}\right\rangle-\left\langle Y_{i}\right\rangle^{2}\right)},
$$

where the mean clock protein concentration $\bar{Y}$ is

$$
\bar{Y}=\frac{1}{N} \sum_{i=1}^{N} Y_{i}
$$

A fully desynchronized state results in $R_{\mathrm{syn}}=0$, whereas complete synchronization in the case of identical oscillators corresponds to $R_{\mathrm{syn}}=1$. The parameters are used as per Ref. 121.

Using this definition, Fig 13(a) shows a transition from a nonsynchronized (arrhythmic, $R_{\text {syn }} \approx 0$ ) to a synchronized (rhythmic, $R_{\text {syn }} \approx 1$ ) state as the deterministic coupling $Q$ increases, for all diversities $\sigma_{\tau}$. As the diversity $\sigma_{\tau}$ increases, the transition point shifts to higher coupling strengths. Results are also presented in Fig. 13(b) by the period of the mean field $T$, which decreases with increasing diversity strength.

\subsubsection{The mean field under stochastic light conditions - stochastic coherence}

Light affects circadian oscillators and under natural day-night conditions leads to externally driven entrainment. Following Díez-Noguera, ${ }^{120}$ it is assumed that light 


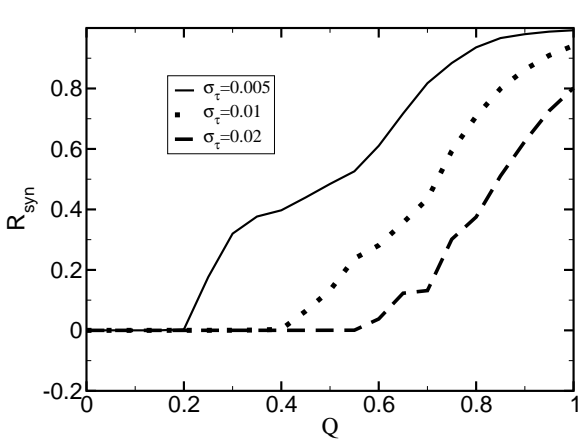

(a)

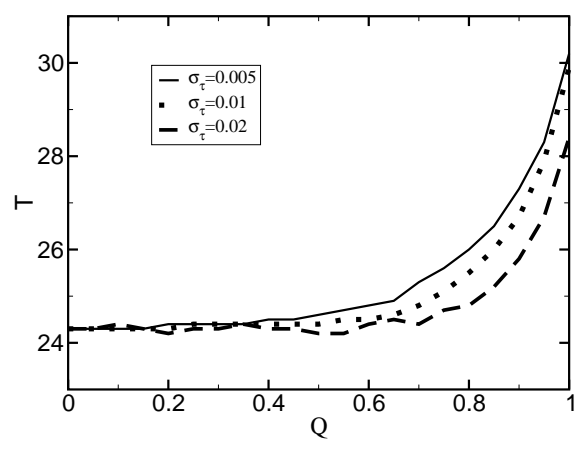

(b)

Fig. 13. Transition from a nonsynchronized to a synchronized state by increasing coupling $Q$ in an ensemble of $N=10,000$ Goodwin oscillators with Gaussian distributed diversity in the absence of noise. Plots showing (a) the synchronization degree $R_{\text {syn }}$ versus the coupling parameter $Q$ for various values of the diversity coefficient $\sigma_{\tau},(\mathrm{b})$ the period of the mean field $T$, calculated from the main peak of the power spectrum versus the coupling parameter $Q$ for various values of the diversity coefficient $\sigma_{\tau}$.

has an inhibitory influence on the coupling strength between the cellular oscillators. Ullner et al. ${ }^{121}$ assume a direct relation between light and coupling $Q$, and represent a random illumination by $Q=Q_{0}+\zeta(t)$. The global noise term $\zeta(t)$ is Gaussian, with zero mean and intensity $\sigma_{m}^{2}$ defined by the correlation $\langle\zeta(t) \zeta(t+\tau)\rangle=\sigma_{m}^{2} \delta(\tau)$. This noise is multiplicative, due to its dependence on the state variable representing the external signaling molecule concentration, $F$. In addition, it is assumed to be global because light affects all clock cells similarly.

One of the mechanisms through which stochastic coherence arises is through a noisy precursor area of a Hopf bifurcation. ${ }^{126,127}$ Therefore, the value of the coupling strength $Q_{0}$ is fixed just before the onset of the synchronization transition within the arrhythmic regime. Under these conditions, random fluctuations in the coupling (i.e., in the external light) allow the cells to temporarily and rapidly surpass the bifurcation onset and move the system into the rhythmic regime, enhancing the stochastic coherence effects. On the other hand, for large couplings $(Q>0.7)$ the period of the mean field $T$ depends strongly on $Q$ [Fig. 13(b)]. This is destructive for stochastic coherence, since the dynamical variability in large $Q$ leads to a large variability of the period of the mean response and thus to the reduction of its coherence. Hence, this range of coupling values should be avoided when looking for stochastic coherence.

As shown in Fig. 13, deterministic synchronization depends on the diversity $\sigma_{\tau}$ and hence $Q_{0}$ should also be chosen with respect to it. Noise-induced coherence is expressed by a bell-shaped curve of the coherence measure with respect to the noise intensity $\sigma_{m}^{2}$ of the global noise $\zeta(t)$ (Fig. 14). The coherence level is given by the decay time of the autocorrelation function of the mean field, for increasing global noise intensity $\sigma_{m}^{2}$ and small rescaling diversity $\sigma_{\tau}=0.005$. Whereas 


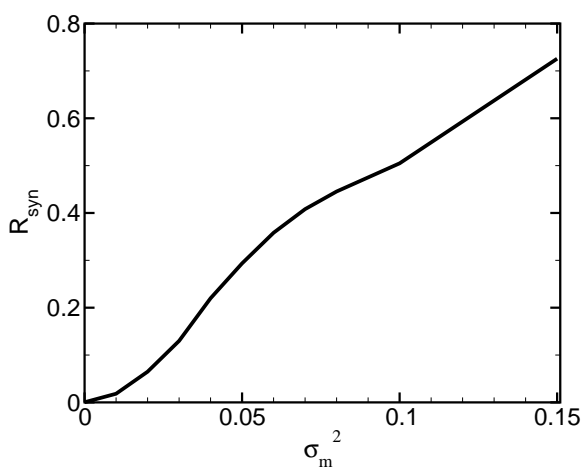

(a)

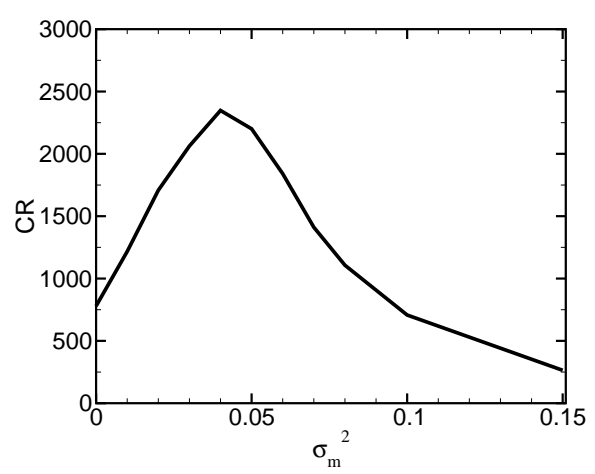

(b)

Fig. 14. Effect of stochasticity seen in (a) synchronization coefficient $R_{\text {syn }}$ versus noise intensity $\sigma_{m}^{2}$, (b) coherence level CR versus noise intensity $\sigma_{m}^{2}$ in a normally distributed ensemble of 10,000 Goodwin oscillators with fixed coupling $Q_{0}=0.15$ and $\sigma_{\tau}=0.005$. Other parameters are as given in literature. ${ }^{121}$

synchronization measured by $R_{\text {syn }}$ increases monotonically with the noise intensity $\sigma_{m}^{2}$, the coherence shows a clear resonance-like behavior with a well pronounced maximum at an optimal noise level of $\sigma_{m, \text { opt }}^{2} \approx 0.04$. It can be seen that an increasing amount of global noise synchronizes the oscillators [Fig. 14(a)], which initially leads to an increase in the coherence of the mean field [Fig. 14(b)]. As the noise intensity increases further, however, synchronization keeps increasing (since all oscillators are driven globally by the noise) but the coherence of the signal decays. Thus, coherence and synchronization are different effects: synchronization is necessary but not sufficient for the emergence of coherence. This leads to stochastic coherence, which can be qualitatively seen in the dynamics of the mean field but not on that of the individual oscillators. It is interesting to note that noiseimproved coherence occurs even in the case of weak synchronization, i.e., even when the individual genetic oscillators still have large dynamical variations amongst them.

In conclusion, in the absence of noise, the amplitude of the oscillations in the mean field is very small. The individual cells are oscillating, but in an incoherent way. Stronger noise enhances the coupling, and so increases the amplitude of the mean field rhythm. The optimal noise intensity arises as a compromise between a strong coupling (and hence enhanced synchronization and large amplitude of the mean field rhythm), and a low destructive influence of the stochastic fluctuations, which reduce the coherence. For large noise levels, the coherence of the individual oscillators becomes dominated by the noise fluctuations, and the resulting loss of regularity is also reflected in the global rhythm. A detailed description of the phenomena and the dynamics of the mean field and the individual oscillators for different noise intensities can be found in Ref. 121. 


\section{Speed Dependent Cellular Decision Making (SdCDM) in Noisy Genetic Networks}

Cellular decision making is a nonlinear process through which cells decide between functionally different fates such as cell differentiation, survival, apoptosis and selfrenewal. ${ }^{131,132}$ One study, which will be reviewed here, looks into the mechanism of Speed dependent Cellular Decision Making (SdCDM), which is the dependence of cellular decision making on the speed with which external signals reach steady state, referred to as the signal speed, and associated fluctuations. ${ }^{37-39}$ This effect was tested on low and high-order switches which act as bistable systems.

\subsection{Speed dependent Cellular Decision Making in a small genetic switch}

In order to examine the SdCDM effect on small networks, a genetic switch [Fig. 15(a)] consisting of two transcription factors $X$ and $Y$ was used. ${ }^{37}$ The switch is exposed to two external signals $S_{1}$ and $S_{2}$ that represent protein kinases. These kinases convert each transcription factor to its active phosphorylated form $\left(X^{a}\right.$ or $\left.Y^{a}\right)$, which enhances the production of its own gene and inhibits the expression of

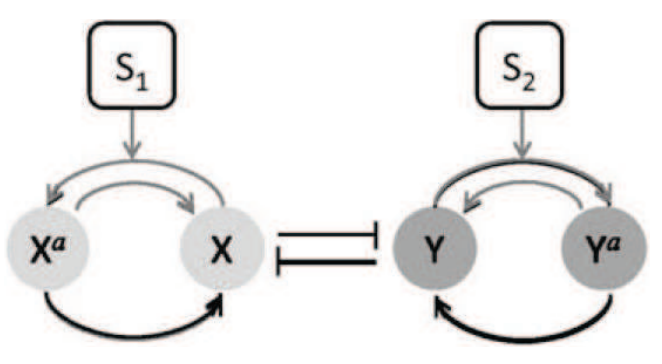

(a)

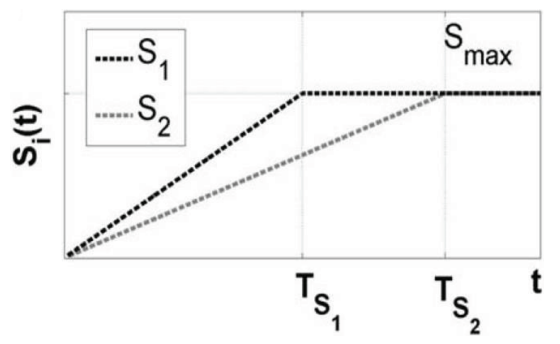

(b)

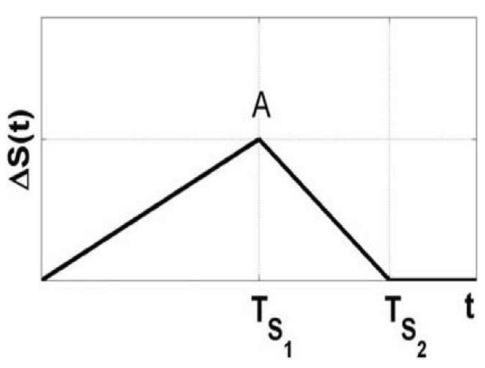

(c)

Fig. 15. Small toggle switch network with external signals which have different growth times. (a) Diagram showing transcriptional small toggle switch. The switch consists of two transcription factors $X$ and $Y$ and their phosphorylated forms $X^{a}$ and $Y^{a}$ under the effect of two external signals $S_{1}$ and $S_{2}$, (b) time evolution of the external signals $S_{1}$ and $S_{2}$ with different growth times $T_{S_{1}}$ and $T_{S_{2}}$ but equal steady states at $S_{\max }=10,(\mathrm{c})$ the asymmetry $\Delta S(t)$ between the external signals, which is the difference in signal strength $S_{1}(t)-S_{2}(t)$ with a maximum asymmetry of $A$. 


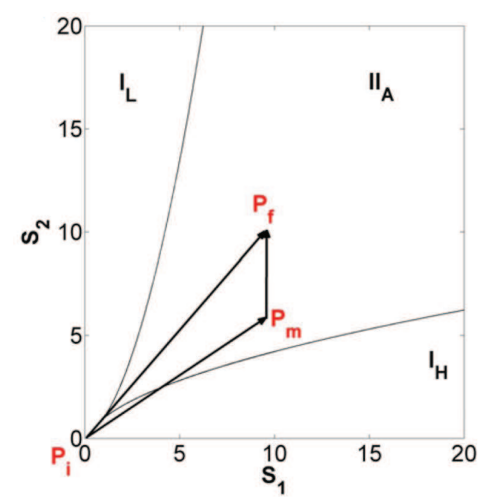

(a)

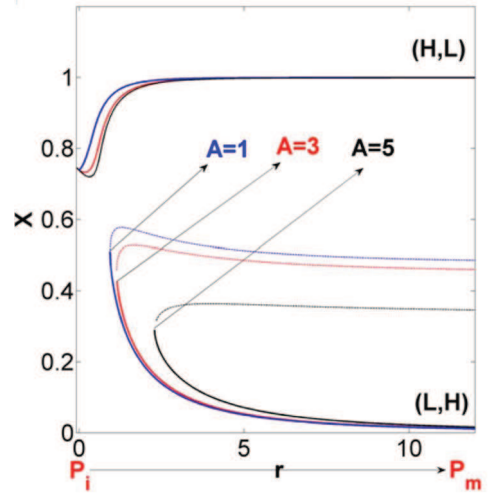

(b)

Fig. 16. Bifurcation analysis of the small genetic switch. (a) Two-dimensional phase-space diagram for protein $X$ in the space $\left(S_{1}, S_{2}\right)$ with $I_{H, L}$ being the regions of monostability with high or low values of $X$, respectively, and $I I_{A}$ being the region of bistability. The direct path from $P_{i} \rightarrow P_{f}$ gives a symmetric supercritical bifurcation diagram, while the indirect path $P_{i} \rightarrow P_{m} \rightarrow P_{f}$ gives asymmetry, (b) asymmetric bifurcation diagram of $X$, where $r$ is the distance between $P_{i}$ and a point along the path $P_{i} \rightarrow P_{m}$. $(H, L)$ represents the variables $X$ and $Y$ having high and low values, respectively. Unitless parameter values are given in Ref. 37.

the other gene. In the system, the signals [Fig. 15(b)] have different growth times $T_{S_{1}}$ and $T_{S_{2}}$, but saturate to equal steady states at $S_{\max } \cdot{ }^{37}$ The transient asymmetry $\Delta S(t)$, between the two external signals reaches a maximum asymmetry $A$ [Fig. 15(c)] and is defined as $\max (\Delta S(t))=S_{\max }\left(1-\left(T_{S_{1}} / T_{S_{2}}\right)\right)$.

\subsubsection{Bifurcations under the effect of external signals with different growth times}

Figure 16(a) shows the different stability regions in the parameter space $\left(S_{1}, S_{2}\right)$. The borders separate the regions of monostability $I_{H}$ (where $X>Y$ has high values) and $I_{L}$ (vice versa) from the region of bistability $I I_{A}$, where $X$ and $Y$ have high and low concentrations, respectively or vice-versa. The transition from monostability to bistability represents a decision making process, where the cell moves from an initial monostable state $P_{i}$ to its final bistable state $P_{f}$, at which $t=T_{S_{2}}$. The path $P_{i} \rightarrow P_{f}$ (which represents the external signals $S_{1}$ and $S_{2}$ having the same growth times) gives a symmetric supercritical pitchfork bifurcation without any distortion.

On the other hand, following path $P_{i} \rightarrow P_{m} \rightarrow P_{f}$ (which represents $S_{1}$ and $S_{2}$ having different growth times), where $t=T_{S_{1}}$ at $P_{m}$, the symmetry in the supercritical pitchfork bifurcation diagram is broken. By increasing the maximum asymmetry $A$, the bifurcation delay, which is the time needed to leave the neighborhood of the unstable branch in the bifurcation diagram, was decreased [Fig. 16(b)]. ${ }^{37}$ Throughout bifurcation analysis, in order to concentrate on the effects of external 


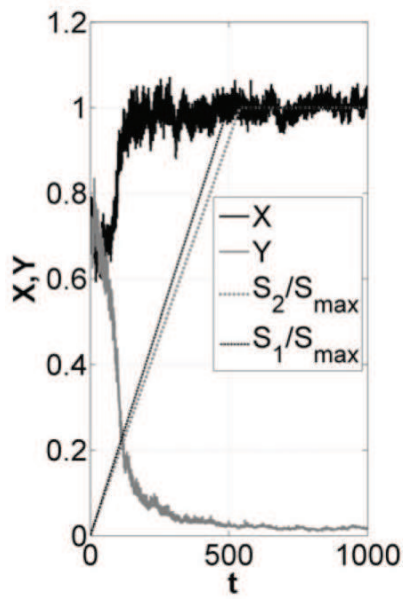

(a)

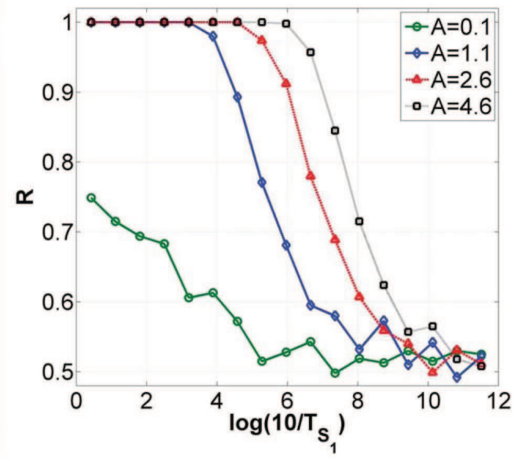

(b)

Fig. 17. Selectivity strength under different growth speeds of external signals in a toggle switch. (a) Typical time evolution of concentrations of $X$ and $Y$. The value of $X$ increases and levels out at 1, while the trajectory of $Y$ goes to zero, (b) the probability $R$, of reaching the upper attractor $(X, Y)=(H, L)$ for different values of maximal asymmetry $A$. As the growth speed of $T_{S_{1}}$ is increased, the probability $R$ tends to 0.5 . Parameter values are stated in Ref. 37 .

signals on genetic switch dynamics, the values of all parameters except $S_{1}$ and $S_{2}$, were taken to be symmetric and constant. ${ }^{37}$

\subsubsection{Effect of different stochastic growth speeds of external signals}

Given different growth times for the external signals $S_{1}$ and $S_{2}$ and parameters as defined in Ref. 37, the trajectories of the factors $X$ and $Y$ tended towards the attractor $(X, Y)=(1,0)$ [Fig. 17(a)]. This corresponds to the upper branch of the bifurcation diagram [Fig. 16(b)] and results from the fact that $S_{1}$ reaches steady state faster than $S_{2} \cdot{ }^{37}$ The attractors of genetic networks can be taken to correspond to specific gene expression programs and consequently, to cell types or cell fates. ${ }^{133}$

To test the effect of the growth speed of external signals in the presence of noise, different values of $T_{S 1}$ were inserted in $S_{1}(t)=\left(S_{\max } / T_{S 1}\right) t$, and $T_{S 2}$ was varied according to $T_{S 2}=\left(S_{\max } / S_{\max }-A\right) T_{S 1}$, keeping $A$ and $S_{\max }$ constant. Thus, the speed was varied but the decision path, $P_{i} \rightarrow P_{m} \rightarrow P_{f}$, remained fixed. ${ }^{37}$ As the growth speed $T_{S_{1}}$ was decreased [Fig. 17(b)], the probability $R$ of reaching an attractor in the upper branch of the bifurcation path tend to 0.5 , which implies symmetry between the upper and lower attractors in the bifurcation diagram, nullifying the bias produced by the external signals. Consequently, high growth speeds, $T_{S_{1}}$ allow for a stronger selection process, even in the presence of noise. In addition, a high maximum asymmetry $A$, gives a higher value for probability of bias $R$ and hence, a high bias in favor of the upper attractor [Fig. 17(b)]. 


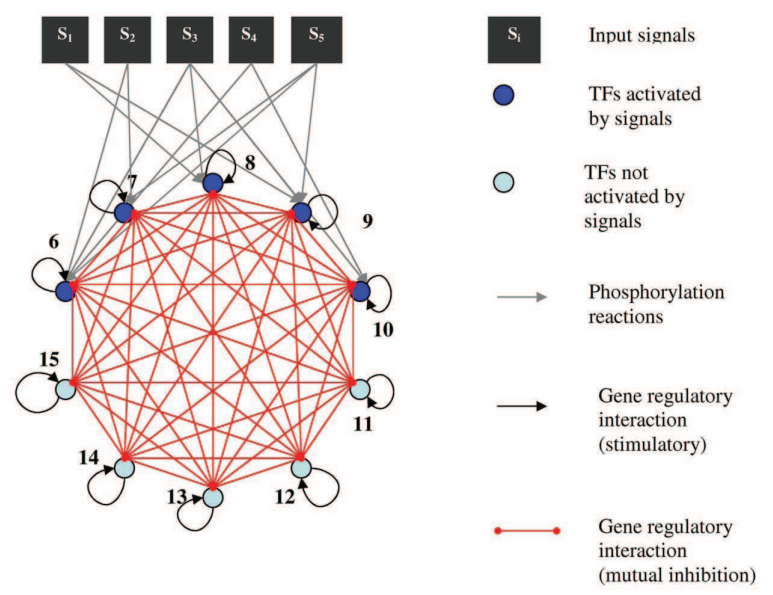

Fig. 18. High-dimensional genetic toggle switch network which consists of two sets of nodes representing transcription factors $\mathrm{TF}_{i}$ with self-activation and cross-inhibition reactions. The former includes the nodes 6-10, and their activation is affected by five external signals $S_{1}, S_{2}, S_{3}, S_{4}$ and $S_{5}$. The latter involves the nodes 11-15, which do not have a direct effect from the external signals.

\subsection{Speed dependent Cellular Decision Making in large genetic networks}

This section generalizes the effects of SdCDM in high-dimensional circuits. ${ }^{38}$ In this case, the circuit (Fig. 18) consists of two layers of self-activating and mutuallyinhibiting transcription factors $\mathrm{TF}_{i}$ represented by nodes. There are five external signals $S_{1}, S_{2}, S_{3}, S_{4}, S_{5}$ that trigger phosphorylation reactions. Each signal has a different growth time but saturates to the same maximal strength and affects only a number of the outer nodes. ${ }^{38}$

\subsubsection{The role of growth speed in decision making}

When $S=S_{1}=S_{2}=S_{3}=S_{4}=S_{5}$, a subcritical pitchfork bifurcations occurs due to the disconnection of emerging branches in the bifurcation diagrams. ${ }^{38}$ In order to analyze whether results noted for small networks can be replicated, i.e., whether different signal strengths and timings affect attractor selection, two pairs of inputs, which will be denoted by $\left(I_{15}, I_{75}\right)$ and $\left(I_{75}, I_{94}\right)$, that lead to the same attractor were chosen. ${ }^{38}$ Each input takes the general form $I_{k}(t)=\left(S_{1}(t), S_{2}(t), S_{3}(t), S_{4}(t), S_{5}(t)\right)_{k}$, where all signals $S_{i}$ have the same initial and final amplitudes but different growth times [Fig. 19(a)]. The inter-trajectory distance (ITD) is a metric of the distance between two trajectories induced, respectively by signals $I_{k}$ and $I_{k^{\prime}}$. It is defined as:

$$
\operatorname{ITD}_{\left(I_{k}, I_{k^{\prime}}\right)}(t)=1-r_{\left(I_{k}, I_{k^{\prime}}\right)}(t),
$$



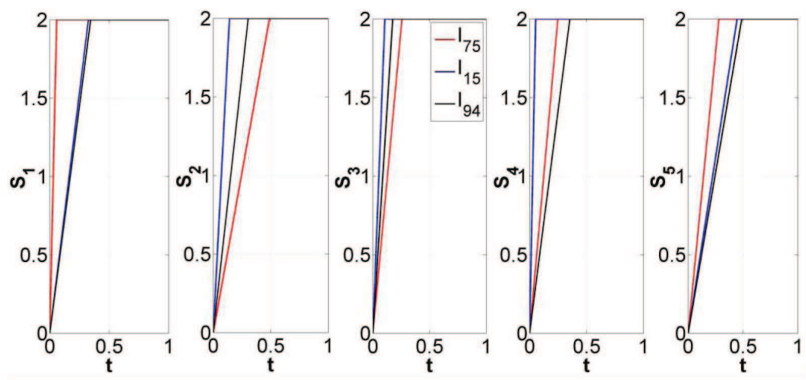

(a)

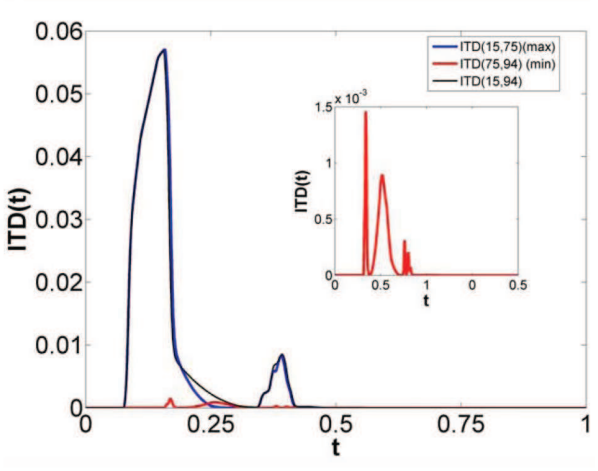

(b)

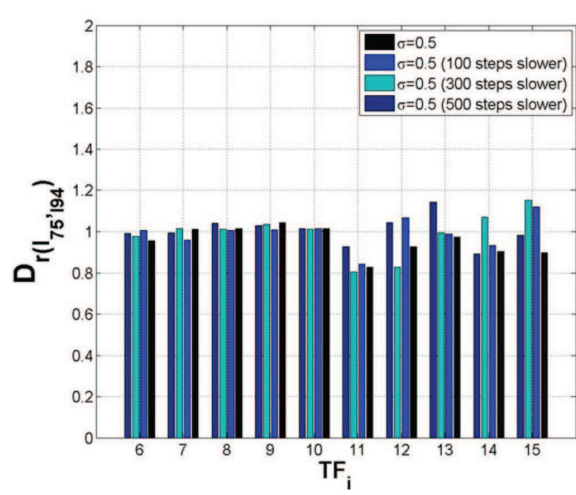

(c)

Fig. 19. (Color online) External signals' profiles and the effect of speed on high-dimensional toggle switches. (a) Time evolution of each signal $S_{i}$ for $i=1, \ldots, 5$ for three different sets of inputs $I_{15}, I_{75}$ and $I_{94}$, (b) time evolution of the inter-trajectory distance $\operatorname{ITD}(t)$ for pairs of the inputs that lead to the same end attractor. The inset shows the trajectory of the pair $\left(I_{75}, I_{94}\right)$ magnified, (c) the distance between final distributions $D_{r}$ for each node $\mathrm{TF}_{i}$ with $i=6, \ldots, 15$. The distance occurs from the pair of inputs $\left(I_{75}, I_{94}\right)$. The legend describes four cases where the inputs are the same but signals $S_{i}$ are 100, 300 and 500 numerical integration time-steps slower and noise $\sigma=0.5$.

where $r_{\left(I_{k}, I_{k^{\prime}}\right)}(t)$ is the correlation between the trajectories induced by inputs $I_{k}$ and $I_{k^{\prime}}$. Results have shown that the largest inter-trajectory distance is between $I_{15}$ and $I_{75}$, while $I_{75}$ and $I_{94}$ give the smallest distance [Fig. 19(b)]. Furthermore, the distance between final probability distributions of each attractors was computed through the formula $D_{r\left(I_{k}, I_{k^{\prime}}\right)}=1-r_{\left(I_{k}, I_{k^{\prime}}\right)}$, where $r_{\left(I_{k}, I_{k^{\prime}}\right)}$ is the correlation between the distributions across attractors. Symmetry corresponds to a smaller distance $D_{r}$ between the final distributions. Decreasing growth speed with a high value of noise increases the distance $D_{r}$, as seen in Fig. 19(c) for inputs $\left(I_{75}, I_{94}\right)$, which implies that decreasing growth speed increases the asymmetry. This agrees with previous observations that a decrease in signal growth speed leads to more specific attractor selectivity. However, this is true to a lesser extent, depending on 
the trajectories being analyzed. ${ }^{38}$ Thus, whereas the observation holds for the pair $\left(I_{75}, I_{94}\right)$ when the ITD is small, the pair $\left(I_{15}, I_{75}\right)$ which lead to the same attractor but have a larger ITD induces the opposite effect (results not shown). The impact of noise in increasing growth speed symmetry can be seen in Ref. 38 .

\section{Discussion}

In this review, four different phenomena found in natural biological systems have been mathematically modeled via simple synthetic genetic networks in order to discuss and explain emergent, unexpected and complex dynamics in relatively simple network systems. The first phenomenon was cell-to-cell communication in a population of synthetic genetic repressilator networks coupled via phase-attractive or phase-repulsive quorum sensing. ${ }^{2,54-56,58-61}$ The coupling system used was the Lux system, ${ }^{56}$ borrowed from nature. The analysis focused on how different phaserepulsive coupling strengths led to different dynamical regimes, such as oscillations, inhomogeneity, steady states and chaos. The second study discussed also looked at repressilators with phase repulsive quorum sensing, but focused on how these interact together in large populations. In agreement with small repressilator systems, the selection of coupling strength lead to either regulated clustering (of oscillations or steady states) or chaotic behavior. In both small and large populations, as the coupling strength was increased, the system was observed to go from oscillatory, to inhomogeneous limit cycles, inhomogeneous and homogeneous steady states, and chaotic regimes. The latter is thought to play a role in high stress situations by allowing for a system's adaptability, and hence, survival. There is also a possibility of fine-tuning protein levels via the system size and coupling strength selection. This can be of paramount importance within a bioindustry and therapeutics setting. This system can be used as an alternative and much simpler mode of studying cell differentiation. More specifically, it can be used as a tool when looking at community traits in artificial cell differentiation, which is a mathematical concept wherein synchronized and identical cells persist in exhibiting a range of dynamics.

In the third case, the phenomenon analyzed was noise, and how it can have constructive effects on a system. The authors used a Goodwin oscillator, ${ }^{46,51}$ representative of the circadian clock. They showed that within an artificial constant-light setting, an arrhythmic system which is on the brink of coherent oscillations can be pushed into this regime through the presence of noise. ${ }^{121}$ Thus, noise effectively served to broaden the parameter range within which synchronized and sustained oscillations occur. This review also informed on possible synchronization factors, such as light profiles which can lead to externally-driven entrainment. ${ }^{128-130}$ This demonstrates how extra-cellular factors have a potential to be used as triggers in genetic networks.

In the fourth case, a bistable system was used to analyze decision making in natural systems found in differentiation, apoptosis and survival mechanisms, among many others. ${ }^{131,132}$ The authors have shown how cellular decision making can be 
biased via incoming external signals. ${ }^{37-39}$ More specifically, the speed with which external signals reached steady states and the transient maximal asymmetry between these signals could bias the system towards a specific attractor. The authors also showed how the impact of the phenomenon of SdCDM decreases with increasing system size. ${ }^{38}$

The analysis carried out has demonstrated how mathematical analytical techniques via synthetic biology can be used to elucidate novel information on the causes and effects of observed dynamics in genetic networks, which would otherwise be more difficult to understand via purely experimental methods. These studies highlight the role which mathematics should play in synthetic and systems molecular biology. Throughout the review, a link is made between mathematical analysis and experimental work to highlight how the different fields can work in tandem. This was done by analyzing parameters and using settings and conditions which can be set up relatively easy within an experimental setting.

In a competitive environment, such as the bio-industry, the drive for detailed, fast, cost-effective and informative results will continue to increase. Analytical modeling of simple and complex synthetic networks will allow us to account for the unexpected and complex dynamics resulting from nonlinear and nonequilibrium systems. We have showcased that in-depth analysis of assemblies of networks should be incorporated into genetic regulatory network studies in order to understand and control emergent properties. This will contribute towards the development of synthetic biology as a robust and predictable field in biological engineering.

\section{Acknowledgments}

EU's work is supported by the Scottish Universities Life Sciences Alliance (SULSA). AZ acknowledges support from the CR-UK, Eve Appeal funded project PROMISE2016, the Deanship of Scientific Research, King Abdulaziz University, Jeddah, grant No. (20/34/Gr) and the Russian Foundation for Basic Research (14-02-01202). YB's work is supported by the Malta Government Scholorship Scheme, UCL Department of Mathematics, the UK Earth and Physical Sciences Research Council (EPSRC) and UCL Department of Biochemical Engineering.

\section{References}

1. T. S. Gardner, C. R. Cantor and J. J. Collins, Nature 403, 6767 (2000).

2. M. B. Elowitz and S. Leibler, Nature 403, 6767 (2000).

3. J. Stricker et al., Nature 456, 7221 (2008).

4. M. Tigges et al., Nature 457, 7227 (2009).

5. T. Danino et al., Nature 463, 7279 (2010).

6. E. Fung et al., Nature 435, 7038 (2005).

7. J. Kim and E. Winfree, Mol. Syst. Biol. 7, 465 (2011).

8. M. R. Atkinson et al., Cell 113, 5 (2003).

9. A. E. Friedland et al., Science 324, 5931 (2009).

10. S. Basu et al., Nature 434, 1130 (2005). 
Y. Borg et al.

11. C. T. Fernando et al., J. R. Soc. Interface 6, 34 (2009).

12. A. Levskaya et al., Nature 438, 7067 (2005).

13. H. Ye et al., Science 332, 6037 (2011).

14. J. J. Tabor, A. Levskaya and C. A. Voigt, J. Mol. Biol. 405, 2 (2011).

15. J. C. Anderson, C. A. Voigt and A. P. Arkin, Mol. Syst. Biol. 7, 133 (2007).

16. A. Tamsir, J. J. Tabor and C. A. Voigt, Nature 469, 7329 (2011).

17. A. Goni-Moreno and M. Amos, BMC Syst. Biol. 6, 126 (2012).

18. H. Kobayashi et al., Proc. Natl. Acad. Sci. USA 101, 22 (2004).

19. J. Bonnet et al., Science 340, 6132 (2013).

20. P. Siuti, J. Yazbek and T. K. Lu, Nat. Biotechnol. 31, 5 (2013).

21. C. M. Ajo-Franklin et al., Genes Dev. 21, 18 (2007).

22. T. K. Lu, A. S. Khalil and J. J. Collins, Nat. Biotechnol. 27, 12 (2009).

23. A. S. Khalil and J. J. Collins, Nat. Rev. Genet. 10, 367 (2010).

24. W. C. Ruder, T. Lu and J. J. Collins, Science 333, 6047 (2011).

25. W. Weber and M. Fussenegger, Nat. Rev. Genet. 13, 1 (2011).

26. E. Andrianantoandro et al., Mol. Syst. Biol. 2, 2006.0028 (2006).

27. L. A. Moran et al., Biochemistry, 2nd edn. (Neil Patterson Publishers, Prentice Hall, 1994).

28. T. Liu and J. Borjigin, J. Biol. Rhythms. 20, 5 (2005).

29. M. F. Crowley and I. R. Epstein, J. Phys. Chem. 93, 2496 (1989).

30. K. Kaneko and T. Yomo, Physica D 75, 89 (1994).

31. P. Hänggi, Chem. Phys. Chem. 3, 3 (2002).

32. E. I. Volkov, E. Ullner and J. Kurths, Chaos 15, 023105 (2005).

33. H. Haken, Synergetics (Springer, Berlin, 1977).

34. G. Nicolis and I. Prigogine, Self-Organization in Nonequilibrium Systems (WileyBlackwell, New York, 1977).

35. F. Sagus, J. M. Sancho and J. García-Ojalvo, Rev. Mod. Phys. 79, 829 (2007).

36. M. Tigges et al., Nature 457, 7227 (2009).

37. N. R. Nene, J. García-Ojalvo and A. Zaikin, PLoS ONE 7, e32779 (2012).

38. N. R. Nene and A. Zaikin, PLoS ONE 7, e40085 (2012).

39. N. R. Nene and A. Zaikin, Phys. Rev. E 87, 012715 (2013).

40. J. B. Gurdon, P. Lemaire and K. Kato, Cell 75, 5 (1993).

41. Y. Saka et al., BMC Syst. Biol. 5, 54 (2011).

42. A. Koseska et al., Phys. Rev. E 75, 031916 (2007).

43. R. Laje and G. B. Mindlin, Phys. Rev. Lett. 89, 288102 (2002).

44. C. R. McClung, Am. Soc. Plant Biol. 18, 4 (2006).

45. M. B. Miller and B. L. Bassler, Annu. Rev. Microbiol. 55, 165 (2001).

46. B. C. Goodwin, Temporal Organization in Cells. A Dynamic Theory of Cellular Control Processes (Academic Press, London, UK, 1963).

47. C. H. Ko et al., PLoS Biol. 8, 10 (2010).

48. M. Cohen et al., Dev. Cell 19, 1 (2010).

49. F. Murray-Zmijewski, E. A. Slee and X. Lu, Nat. Rev. Mol. Cell Biol. 9, 9 (2002).

50. E. Ullner et al., Int. J. Mod. Phys. B 26, 1246009 (2012).

51. O. Purcell et al., J. R. Soc. Interface 7, 52 (2010).

52. J. B. Hogenesch and H. R. Ueda, Nat. Rev. Genet. 12, 6 (2011).

53. A. Pokhilko et al., Mol. Syst. Biol. 8, 574 (2012).

54. J. García-Ojalvo, M. B. Elowitz and S. H. Strogatz, Proc. Natl. Acad. Sci. USA 101, 30 (2004).

55. D. McMillen et al., Proc. Natl. Acad. Sci. USA 99, 2 (2002).

56. L. You et al., Nature 428, 6985 (2004). 
57. E. I. Volkov and M. N. Stolyarov, Phys. Lett. A 159, 61 (1991).

58. S. K. Han, C. Kurrer and Y. Kuramoto, Phys. Rev. Lett. 75, 17 ( 1995).

59. G. Balzsi et al., Phys. Rev. E 64, 041912 (2001).

60. E. Ullner et al., Phys. Rev. Lett. 99, 14 (2007).

61. A. Koseska et al., J. Theor. Biol. 263, 2 (2010).

62. E. Ullner et al., Phys. Rev. E 78, 031904 (2008).

63. L. Glass and M. C. Mackey, From Clocks to Chaos: The Rhythms of Life (Princeton University Press, Princeton, NJ, 1988).

64. H. Meinhardt, Models of Biological Pattern Formation (Academic, New York, 1982).

65. E. Ullner et al., in Handbook on Biological Networks, eds. S. Boccaletti, V. Latora and Y. Moreno (World Scientific Publishing Company, Singapore, 2009), pp. 33-58.

66. P. Swain, M. Elowitz and E. Siggia, Proc. Natl. Acad. Sci. USA 99, 20 (2002).

67. P. Swain and A. Longtin, Chaos 16, 2 (2006).

68. J. P. Eckmann and D. Ruelle, Rev. Mod. Phys. 57, 617 (1985).

69. I. P. Mariño, E. Ullner and A. Zaikin, PLoS ONE 8, e79892 (2013).

70. J. Slipantschuk et al., Chaos 20, 045117 (2010).

71. D. Golomb et al., Phys. Rev. A 45, 6 (1992).

72. K. Okuda, Physica D 63, 424 (1993).

73. K. Miyakawa and K. Yamada, Physica D 151, 217 (2001).

74. W. Wang, I. Z. Kiss and J. L. Hudson, Phys. Rev. Lett. 86, 21 (2001).

75. I. Z. Kiss and J. L. Hudson, Chaos 13, 3 (2003).

76. A. Kuznetsov and J. Kurths, Phys. Rev. E 66, 026201 (2002).

77. S. C. Manruiba and A. S. Mikhailov, Phys. Rev. E 60, 1579 (1999).

78. G. Osipov, J. Kurths and C. Zhou, Synchronization in Oscillatory Networks (Springer, Berlin, 2007).

79. W. Wang, I. Z. Kiss and J. L. Hudson, Chaos 10, 1 (2000).

80. E. I. Volkov and M. N. Stolyarov, Biol. Cybern. 71, 4 (1994).

81. J. Paulsson and M. Ehrenberg, Q. Rev. Biophys. 34, 1 (2001).

82. K. Kaneko, Physica D 41, 137 (1990).

83. N. Suzuki, C. Furusawa and K. Kaneko, PLoS ONE 6, e27232 (2011).

84. J. M. Raser and E. K. O'Shea, Science 309, 5743 (2005).

85. N. Maheshri and E. K. O'Shea, Annu. Rev. Biophys. Biomol. Struct. 36, 413 (2007).

86. A. Eldar and M. B. Elowitz, Nature 467, 7312 (2010).

87. B. Munsky, G. Neuert and A. van Oudenaarden, Science 336, 6078 (2012).

88. A. Sanchez, S. Choubey and J. Kondev, Annu. Rev. Biophys. 42, 469 (2013).

89. C. J. Zopf et al., PLOS Comput. Biol. 9, e1003161 (2013).

90. L. Gammaitoni et al., Rev. Mod. Phys. 70, 223 (1998).

91. F. Sagués, J. M. Sancho and J. García-Ojalvo, Rev. Mod. Phys. 79, 829 (2007).

92. W. Horsthemke and R. Lefever, Noise-Induced Transitions (Springer, Berlin, 1984).

93. B. Lindner et al., Phys. Rep. 392, 321 (2004).

94. P. Reimann, Phys. Rep. 361, 57 (2002).

95. K. Wiesenfeld, J. Stat. Phys. 38, 1071 (1985).

96. A. Neiman, P. I. Saparin and L. Stone, Phys. Rev. E 56, 270 (1997).

97. A. S. Pikovsky and J. Kurths, Phys. Rev. Lett. 78, 775 (1997).

98. A. Fraser and J. Tiwari, J. Theor. Biol. 47, 2 (1974).

99. A. F. Tiwari and R. Beckman, J. Theor. Biol. 45, 2 (1974).

100. J. Lewis, Curr. Biol. 13, 16 (2003).

101. D. Bratsun et al., Proc. Natl. Acad. Sci. USA 102, 41 (2005).

102. D. Gonze, J. Halloy and A. Goldbeter, Proc. Natl. Acad. Sci. USA 99, 2 (2002).

103. P. O. Westermark et al., PloS Comp. Biol. 5, 11 (2009). 
104. D. P. King et al., Cell 89, 4 (1997).

105. A. Balsalobre, F. Damiola and U. Schibler, Cell 93, 6 (1998).

106. S. Yamazaki et al., Science 288, 5466 (2000).

107. E. D. Herzog and W. J. Schwartz, J. App. Phys. 92, 1 (2002).

108. M. H. Hastings, A. B. Reddy and E. S. Maywood, Nat. Rev. 4, 8 (2003).

109. D. Granados-Fuentes et al., J. Neurosci. 24, 3 (2004).

110. A. F. Carr and D. Whitmore, Nat. Cell Biol. 7, 3 (2005).

111. T. Cambras et al., Proc. Natl. Acad. Sci. USA 104, 18 (2007).

112. P. Thomas et al., J. Theor. Biol. 335, 222 (2013).

113. S. Yamaguchi et al., Science 302, 5649 (2003).

114. A. J. Carr and D. Whitmore, Nat. Cell Biol. 7, 3 (2005).

115. J. E. Quintero, S. J. Kuhlman and D. G. McMahon, J. Neurosci. 23, 22 (2003).

116. M. H. Hastings and E. D. Herzog, J. Biol. Rhythms. 19, 5 (2004).

117. N. Inagaki et al., Proc. Natl. Acad. Sci. USA 104, 18 (2007).

118. D. Granados-Fuentes et al., J. Neurosci. 24, 3 (2004).

119. H. Ohta, S. Yamazaki and D. G. McMahon. Nat. Neurosci. 8, 3 (2005).

120. A. Díez-Noguera, Am. J. Physiol. Regul. Integr. Comp. Physiol. 267, R1118 (1994).

121. E. Ullner et al., Biophys. J. 96, 9 (2009).

122. E. S. Maywood et al., Curr. Biol. 16, 6 (2006).

123. R. Lutz and H. Bujard, Nucleic Acids Res. 25, 6 (1997).

124. B. C. Goodwin, Adv. Enzyme Regul. 3, 425 (1965).

125. D. Gonze et al., Biophys. J. 89, 1 (2005).

126. A. Neiman, P. I. Saparin and L. Stone, Phys. Rev. E 56, 270 (1997).

127. O. Carrillo et al., Europhys. Lett. 65, 452 (2004).

128. A. Levskaya et al., Nature 438, 7067 (2005).

129. J. J. Tabor, A. Levskaya and C. A. Voigt, J. Mol. Biol. 405, 2 (2011).

130. K. Müller and W. Weber, Mol. BioSyst. 9, 4 (2013).

131. G. Balazsi, A. V. Oudenaarden and J. J. Collins, Cell 144, 6 (2011).

132. J. Peltier and D. V. Schaffer, IET Syst. Biol. 4, 1 (2010).

133. A. Kashiwagi et al., PLoS ONE 1, e49 (2006). 